\title{
Monodromy in Hamiltonian Floer theory
}

\author{
Dusa McDuff*
}

\begin{abstract}
Schwarz showed that when a closed symplectic manifold $(M, \omega)$ is symplectically aspherical (i.e. the symplectic form and the first Chern class vanish on $\pi_{2}(M)$ ) then the spectral invariants, which are initially defined on the universal cover of the Hamiltonian group, descend to the Hamiltonian group $\operatorname{Ham}(M, \omega)$. In this note we describe less stringent conditions on the Chern class and quantum homology of $M$ under which the (asymptotic) spectral invariants descend to $\operatorname{Ham}(M, \omega)$. For example, they descend if the quantum multiplication of $M$ is undeformed and $H_{2}(M)$ has rank $>1$, or if the minimal Chern number is at least $n+1$ (where $\operatorname{dim} M=2 n$ ) and the even cohomology of $M$ is generated by divisors. The proofs are based on certain calculations of genus zero Gromov-Witten invariants. As an application, we show that the Hamiltonian group of the one point blow up of $T^{4}$ admits a Calabi quasimorphism. Moreover, whenever the (asymptotic) spectral invariants descend it is easy to see that $\operatorname{Ham}(M, \omega)$ has infinite diameter in the Hofer norm. Hence our results establish the infinite diameter of Ham in many new cases. We also show that the area pseudonorm - a geometric version of the Hofer norm - is nontrivial on the (compactly supported) Hamiltonian group for all noncompact manifolds as well as for a large class of closed manifolds.
\end{abstract}

Mathematics Subject Classification (2000). 53D40, 53D45, 57R58.

Keywords. Hamiltonian group, Floer theory, Seidel representation, spectral invariant, Hofer norm, Calabi quasimorphism.

\section{Contents}

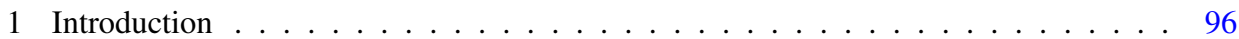

2 Spectral invariants . . . . . . . . . . . . . . . . . . . . 104

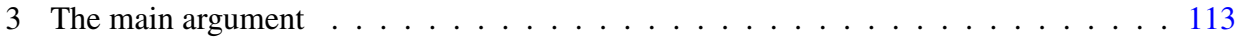

4 Calculations of Gromov-Witten invariants . . . . . . . . . . . . . . . 120

5 Examples . . . . . . . . . . . . . . . . . . . . . . 129

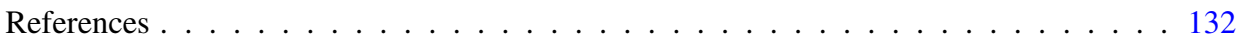

*Partially supported by NSF grant DMS 0604769 . 


\section{Introduction}

Let $(M, \omega)$ be a closed symplectic manifold. Denote by $\operatorname{Ham}:=\operatorname{Ham}(M, \omega)$ its group of Hamiltonian symplectomorphisms and by $\widetilde{\text { Ham }}$ the universal cover of Ham. Each path $\left\{\phi_{t}\right\}_{0 \leq t \leq 1}$ in Ham is the flow of some time dependent Hamiltonian $H_{t}$ and, following Hofer [8], we define its length $\mathscr{L}\left(\left\{\phi_{t}\right\}\right)$ to be:

$$
\mathscr{L}\left(\left\{\phi_{t}\right\}\right)=\int_{0}^{1}\left(\max _{x \in M} H_{t}(x)-\min _{x \in M} H_{t}(x)\right) d t .
$$

The Hofer (pseudo)norm $\|\tilde{\phi}\|$ of an element $\tilde{\phi}=\left(\phi,\left\{\phi_{t}\right\}\right)$ in the universal cover Ham of Ham is then defined to be the infimum of the lengths of the paths from the identity element id to $\phi$ that are homotopic to $\left\{\phi_{t}\right\}$. Similarly we define the norm $\|\phi\|$ of an element in Ham to be the infimum of the lengths of all paths from id to $\phi$. It is easy to see that $\|\phi\|$ is conjugation invariant and satisfies $\|\phi \psi\| \leq\|\phi\|+\|\psi\|$, but harder to see that it is nondegenerate, i.e. $\|\phi\|=0$ iff $\phi=\mathrm{id}$. (This was proved for compactly supported symplectomorphisms of $\mathbb{R}^{2 n}$ by Hofer [8] and for general $M$ by Lalonde-McDuff [14].) It is unknown whether $\|\cdot\|$ is always nondegenerate (and hence a norm) on $\widetilde{\text { Ham }}$ since it may vanish on some elements of the subgroup $\pi_{1}$ (Ham). On the other hand, there is no known counterexample; for some results in the positive direction see Remark 2.9 below.

The question of whether $\|\cdot\|$ is uniformly bounded makes sense even if $\|\cdot\|$ is just a pseudonorm. If is it unbounded on $\widetilde{\mathrm{Ham}}$ or Ham we shall say that this group has infinite (Hofer) diameter. Ostrover [24] showed that $\widetilde{\text { Ham }}$ always has infinite Hofer diameter (we sketch the proof below), while the corresponding result is unknown for Ham in many cases. For example, it is shown in [20] that Ham has infinite diameter when $M$ is a "small" blow up of $\mathbb{C} P^{2}$ but it is unknown whether this remains the case when $M$ is monotone (i.e. the exceptional divisor is precisely one third the size of the line) or is a still bigger blow up.

However, if both $[\omega]$ and $c_{1}(M)$ vanish on $\pi_{2}(M)$ then Schwarz [27] showed that Ham does have infinite diameter. To prove this he established that for such $M$ each element $\tilde{\phi} \in \widetilde{\text { Ham }}$ has a set of so-called spectral invariants

$$
\left\{c(a, \tilde{\phi}) \mid a \in Q H_{*}(M), a \neq 0\right\} \subset \mathbb{R} .
$$

Later, Oh [22], [23] and Usher [29] showed that the numbers $c(a, \tilde{\phi})$ are well defined on $\widetilde{\text { Ham }}$ for all symplectic manifolds. It follows easily from their properties (explained in $\$ 2.2$ below) that $\widehat{\text { Ham }}$ has infinite Hofer diameter. However, they do not in general descend to well defined functions on Ham; in other words it may not be true that $c(a, \tilde{\phi})=c(a, \tilde{\psi})$ whenever $\tilde{\phi}, \tilde{\psi}$ project to the same element of Ham. Schwarz showed that when both $[\omega]$ and $c_{1}(M)$ vanish on $\pi_{2}(M)$ the invariants do descend to Ham. As we explain below, it is then an easy consequence of Ostrover's construction that Ham has infinite diameter. 
Another case in which Ham was known to have infinite diameter is $S^{2}$. The original proof in Polterovich [26] initially appears somewhat different in spirit from the approach presented here, but the arguments in Entov-Polterovich [3] using quasimorphisms bring this result also within the current framework; cf. case (ii) of Theorem 1.3.

In fact there are two questions one can ask here. Do the spectral invariants themselves descend, or is it only their asymptotic versions that descend? Here, following Entov-Polterovich [3], we define the asymptotic spectral invariants $\bar{c}(a, \tilde{\phi})$ for nonzero $a \in Q H_{*}(M)$ by setting

$$
\bar{c}(a, \tilde{\phi}):=\liminf _{k \rightarrow \infty} \frac{c\left(a, \tilde{\phi}^{k}\right)}{k}, \quad \text { for all } \tilde{\phi} \in \widetilde{\text { Ham }} .
$$

Ostrover's construction again implies that Ham has infinite diameter whenever an asymptotic spectral number descends to Ham; see Lemma 2.7.

We shall extend Schwarz's result in two directions, imposing conditions either on $\omega$ via the Gromov-Witten invariants or on $c_{1}$. Recall that the quantum product on $H_{*}(M)$ is defined by the 3-point genus zero Gromov-Witten invariants

$$
\left\langle a_{1}, a_{2}, a_{3}\right\rangle_{\beta}^{M}, \quad \beta \in H_{2}(M), a_{i} \in H_{*}(M),
$$

and reduces to the usual intersection product if these invariants vanish whenever $\beta \neq 0$. In the latter case we shall say that the quantum product (or simply $Q H_{*}(M)$ ) is undeformed. The condition $\left.[\omega]\right|_{\pi_{2}(M)}=0$ is much stronger; in this case there are no $J$-holomorphic spheres at all, and so the quantum product on $M$ is of necessity undeformed. If $\left.[\omega]\right|_{\pi_{2}(M)}=0$, Schwarz's argument easily extends to show that the spectral invariants descend; see Proposition 3.1 (i). However, its generalization in Theorem 1.1 below concerns the asymptotic invariants.

We shall denote by $N$ the minimal Chern number of $(M, \omega)$, i.e. the smallest positive value of $c_{1}(M)$ on $\pi_{2}(M)$. If $\left.c_{1}\right|_{\pi_{2}(M)}=0$ then we set $N:=\infty$. We always denote $\operatorname{dim} M=2 n$. We shall say that $(M, \omega)$ is spherically monotone if there is $\kappa>0$ such that $\left.c_{1}\right|_{\pi_{2}(M)}=\left.\kappa \omega\right|_{\pi_{2}(M)}$ and is negatively monotone if $c_{1}=\kappa[\omega]$ on $\pi_{2}(M)$ for some $\kappa<0$. Recall also that $(M, \omega)$ is said to be (symplectically) uniruled if some genus zero Gromov-Witten invariant of the form $\left\langle\mathrm{pt}, a_{2}, \ldots, a_{m}\right\rangle_{\beta}$, $\beta \neq 0$, does not vanish. It is called strongly uniruled if this happens for $m=3$.

Theorem 1.1. Let $(M, \omega)$ be a closed symplectic manifold such that $Q H_{*}(M)$ is undeformed. Then the asymptotic spectral invariants descend to Ham except possibly if the following three additional conditions all hold: $\operatorname{rank} H_{2}(M)=1, N \leq n$, and $(M, \omega)$ is spherically monotone.

Remark 1.2. (i) The exceptional case does not occur if $M$ has dimension 4. For if $Q H_{*}(M)$ is undeformed then $(M, \omega)$ is minimal. (The class of an exceptional sphere always has nontrivial Gromov-Witten invariant; see [19].) Moreover it follows from 
the results of Taubes-Li-Liu that it cannot be spherically monotone, for if it were it would be uniruled, in particular the quantum product would be deformed. Similarly, the exceptional case does not occur for smooth projective varieties of any dimension. For $N \neq \infty$ implies that at least some nonzero elements in $H_{2}(M)$ are represented by spheres. Hence they must all be (since rank $H_{2}(M)=1$ ). Therefore the conditions imply that $M$ is Fano, and hence, by an argument of Kollar-Ruan that is explained in [10], also symplectically uniruled. It is unknown whether every spherically monotone symplectic manifold is uniruled. If this were true then there would be no exceptions at all.

(ii) Every minimal 4-manifold that is not rational or ruled (such as a $K 3$ surface or a simply connected surface of general type) has vanishing genus zero Gromov-Witten invariants and so is covered by this theorem.

The next result explains what can be proved under various conditions on the minimal Chern number $N$. We denote the even degree homology of $M$ by $H_{\mathrm{ev}}(M)$.

Theorem 1.3. Let $(M, \omega)$ be a closed symplectic $2 n$-dimensional manifold with minimal Chern number $N$. Suppose further that either $H_{\mathrm{ev}}(M)$ is generated as a ring by the divisors $H_{2 n-2}(M)$ or that $Q H_{*}(M)$ is undeformed. Then:

(i) If $N \geq n+1$, the spectral invariants are well defined on Ham except possibly if $N \leq 2 n$ and $(M, \omega)$ is strongly uniruled.

(ii) If $n+1 \leq N \leq 2 n$ the asymptotic spectral invariants are well defined on Ham.

(iii) The conclusion in (ii) still holds if $N=n$ except possibly if $(M, \omega)$ is strongly uniruled or if rank $\mathrm{H}_{2}(M)=1$.

(iv) The conclusion in (ii) also holds when $(M, \omega)$ is negatively monotone, independently of the values of $n, N$.

For example, if $M$ is a 6-dimensional Kähler manifold then $H_{\mathrm{ev}}(M)$ is generated as a ring by $H_{4}(M)$ because $\wedge[\omega]: H^{2}(M) \rightarrow H^{4}(M)$ is an isomorphism. Hence all Calabi-Yau 3-folds satisfy the conditions of this theorem.

Remark 1.4. (i) In Theorem 1.3 the conditions in the second sentence may be replaced by the weaker but somewhat technical condition (D); cf. Definition 3.5.

(ii) Theorem 1.3 is sharp. To see that the non-uniruled hypothesis in (i) is necessary, observe that by Entov-Polterovich [3] the spectral invariants do not descend for $M=\mathbb{C} P^{n}$ although the asymptotic ones do. This condition is also needed in (iii). For example, consider $M=S^{2} \times S^{2}$ which has $N=n=2$ and is strongly uniruled. Ostrover [25] showed that the asymptotic spectral invariants descend if and only if $(M, \omega)$ is monotone, i.e. the two 2 -spheres have equal area. Further, the results do not extend to smaller $N$. Proposition 1.8 below gives many examples of manifolds 
with $N=n-1$ that satisfy the other cohomological conditions but are such that the asymptotic invariants do not descend.

(iii) It is not clear what happens when $N=n$ but $\operatorname{rank} H_{2}(M)=1$. If $(M, \omega)$ is negatively monotone, then by Proposition 3.1 the asymptotic spectral invariants always descend (without any condition on $Q H_{*}(M)$ ), but it is not clear what happens in the positive case. (Cf. the similar missing case in Theorem 1.1.) If $(M, \omega)$ were also projective then $(M, \omega)$ would be uniruled and one would not expect the invariants to descend but in the general case considered here all we can say is that our methods fail. The relevant part of the proof of Proposition 3.1 fails for $N=n$ and $\kappa>0$, while the argument in Lemmas 4.3 and 4.4 definitely needs rank $H_{2}(M)>1$.

(iv) The example of $S^{2} \times S^{2}$ in Remark 1.4(ii) above suggests that perhaps the asymptotic spectral invariants descend for all monotone manifolds. But this is not true. Consider, for example the monotone one point blow up of $\mathbb{C} P^{2}$ with its obvious $T^{2}$ action. It is easy to see that there are circles in $T^{2}$ that represent elements $\gamma \in \pi_{1}(\mathrm{Ham})$ for which $\bar{c}(\mathbb{1}, \gamma) \neq 0$; see [20], [25].

Corollary 1.5. If $(M, \omega)$ satisfies any of the conditions in Theorems 1.1 and 1.3 , then Ham has infinite Hofer diameter.

Proof. This holds by Lemma 2.7 .

Of course, one expects Ham always to have infinite Hofer diameter, but this question seems out of reach with current techniques. However there are other ways to tackle this question. For example, in [20] we show that a small blow up of $\mathbb{C} P^{2}$ has infinite Hofer diameter even though the spectral invariants do not descend by using an argument based on the asymmetry of the spectral invariants, i.e. the fact that the function $\bar{V}$ of Remark 1.11 does not vanish on $\pi_{1}$ (Ham). Also if $\pi_{1}(M)$ is infinite, one can sometimes use the energy-capacity inequality as in Lalonde-McDuff [15].

Another related problem is the question of when the area pseudonorm

$$
\rho^{+}+\rho^{-}: \text {Ham } \rightarrow \mathbb{R}
$$

defined in [17] is nonzero. Here

$$
\rho^{+}(\phi):=\inf \int_{0}^{1} \max _{x \in M} H_{t} d t,
$$

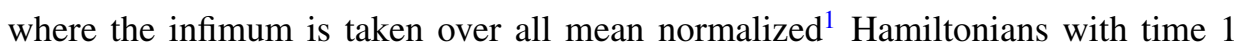
map $\phi$. Further $\rho^{-}(\phi):=\rho^{+}\left(\phi^{-1}\right)$. It is easy to see that $\rho^{+}+\rho^{-}$is a conjugation invariant pseudonorm on Ham. Therefore, because Ham is simple, $\rho^{+}+\rho^{-}$is either

\footnotetext{
${ }^{1}$ I.e. $\int_{M} H_{t} \omega^{n}=0$ for all $t$. Also, this discussion of one sided norms is the one place in this paper where the choice of signs is crucial. In order to be consistent with [17] we shall define the flow of $H_{t}$ to be generated by the vector $X_{H_{t}}$ satisfying $\omega\left(X_{H}, \cdot\right)=-d H_{t}$.
} 
identically zero or is nondegenerate and hence a norm. The difficulty in dealing with it is that one may need to use different lifts $\tilde{\phi}$ of $\phi$ to $\widetilde{\text { Ham }}$ to calculate $\rho^{+}$and $\rho^{-}$. However it has a very natural geometric interpretation. For by [17, Proposition 1.12]

$$
\rho^{+}(\phi)+\rho^{-}(\phi)=\inf (\operatorname{Vol}(P, \Omega) / \operatorname{Vol}(M, \omega))
$$

where the infimum is taken over all Hamiltonian fibrations $(M, \omega) \rightarrow(P, \Omega) \rightarrow S^{2}$ with monodromy $\phi$ around some embedded loop in the base. This ratio is called the area of the fibration $P \rightarrow S^{2}$ since for product fibrations it would be the area of the base.

Since the area pseudonorm $\rho^{+}+\rho^{-}$is never larger than the Hofer norm, the next result also implies Corollary 1.5.

Corollary 1.6. If $(M, \omega)$ satisfies any of the conditions in Theorems 1.1 and 1.3, then the area pseudonorm $\rho^{+}+\rho^{-}$is an unbounded norm on Ham.

Proof. See Lemma 2.8.

This result improves [17, Theorem 1.2] which established the nontriviality of $\rho^{+}+\rho^{-}$only for the cases $\left.\omega\right|_{\pi_{2}(M)}=0$ and $M=\mathbb{C} P^{n}$.

The real problem in understanding the onesided pseudonorms $\rho^{ \pm}$is caused by the possible existence of short loops, i.e. loops in Ham that are generated by Hamiltonians for which $\rho^{+}$(or $\rho^{-}$) is small; see $[17, \S 1.4]$. These are still not understood in the closed case in general, but, as we now explain, it turns out that they cause no problem when $M$ is noncompact.

Let $(M, \omega)$ be a noncompact manifold without boundary, and $U \subset M$ be open with compact closure. Denote by $\operatorname{Ham}^{c} U$ the group of symplectomorphisms generated by functions $H_{t}$ with support in $U$ and by $\widetilde{\operatorname{Ham}}^{c} U$ its universal cover. Denote by $\tilde{\rho}_{U}^{+}$the positive part of the Hofer norm on $\widetilde{\operatorname{Ham}^{c}} U$ and by $\rho_{U}^{+}$the induced function on $\operatorname{Ham}^{c} U$. Notice that in principle $\tilde{\rho}_{U}^{+}$might depend on $U$. But clearly $U^{\prime} \subset U$ implies $\tilde{\rho}_{U^{\prime}}^{+} \geq \tilde{\rho}_{U}^{+}$. A similar remark applies to $\rho_{U}^{+}$.

The following result was suggested by a remark in an early version of [7]. Note that $(M, \omega)$ can be arbitrary here; in particular it need satisfy no special conditions at infinity.

Proposition 1.7. Suppose that $(M, \omega)$ is noncompact and that $U$ is an open subset of $M$ with compact closure. Then for all $H: M \rightarrow \mathbb{R}$ with support in $U$ there is $\delta=\delta(H, U)>0$ such that $\rho_{U}^{+}\left(\phi_{t}^{H}\right)=t \max H$ for all $0 \leq t \leq \delta$. In particular $\rho_{U}^{+}+\rho_{U}^{-}$is a nondegenerate norm on $\operatorname{Ham}^{c} U$.

The proof is given at the end of 2 . Note that $\widetilde{\operatorname{Ham}^{c}} U$ always has infinite Hofer diameter because of the existence of the Calabi homomorphism. However, even 
though $\widetilde{\operatorname{Ham}}^{c} U$ is not a simple group, the kernel of the Calabi homomorphism is simple. Hence the second statement in the above proposition follows from the first because the element $\phi_{t}^{H}$ belongs to this kernel when $\int_{U} H \omega^{n}=0$. For a brief discussion of other issues that arise in the noncompact case, see [20, Remark 3.11].

Finally we describe another class of examples, the one point blow ups. These have $N \mid(n-1)$ but may be chosen to satisfy the other conditions of Theorem 1.3.

Proposition 1.8. (i) Let $M$ be a sufficiently small one point blow up of any closed symplectic manifold $\left(X, \omega_{X}\right)$ such that at least one of $\left[\omega_{X}\right], c_{1}(X)$ does not vanish on $\pi_{2}(X)$. Then the asymptotic spectral invariants do not descend to Ham.

(ii) If $M$ is the one point blow up of a closed symplectic 4-manifold $X$ such that both $\left[\omega_{X}\right]$ and $c_{1}(X)$ vanish on $\pi_{2}(X)$ then the asymptotic spectral invariants do descend to Ham.

The proof is given in $\S 5$.

Corollary 1.9. If $M$ is as in Proposition 1.8 (ii) then Ham supports a nontrivial Calabi quasimorphism.

The proof is contained in the following remark.

Remark 1.10. Other potential applications of these results arise from the work of Entov and Polterovich ([3], [4], [5], [6]). They denote the asymptotic spectral invariant given by an idempotent $e$ in $\mathcal{A}_{M}:=Q H_{2 n}(M)$ by

$$
\mu_{e}: \widetilde{\operatorname{Ham}} \rightarrow \mathbb{R}, \quad \mu_{e}(\cdot):=\bar{c}(e, \cdot)
$$

It is immediate that $\mu_{e}$ descends to Ham iff $\left.\mu_{e}\right|_{\pi_{1}(\mathrm{Ham})}$ vanishes; see Proposition 2.3. The Entov-Polterovich results about nondisplaceablity (see [5] for example) apply whether or not $\mu_{e}$ descends to Ham. But if one is interested in questions about the structure of the Hamiltonian group itself, for example what quasimorphisms or norms it might have or what discrete subgroups it might contain, then our results are relevant.

If $e$ is an idempotent such that $e \mathcal{A}_{M}$ is a field, ${ }^{2}$ then Entov-Polterovich show that $\mu_{e}$ is a homogeneous quasimorphism, i.e. there is $C>0$ such that for all $k \in \mathbb{Z}$ and $\tilde{\phi}, \tilde{\psi} \in \widetilde{\mathrm{Ham}}$

$$
\mu_{e}\left(\tilde{\phi}^{k}\right)=k \mu_{e}(\tilde{\phi}), \quad\left|\mu_{e}(\tilde{\phi} \tilde{\psi})-\mu_{e}(\tilde{\phi})-\mu_{e}(\tilde{\psi})\right| \leq C .
$$

\footnotetext{
${ }^{2}$ When $\mathcal{A}_{M}$ is semisimple, it is tempting to think that $\mu_{e}$ is a quasimorphism for every idempotent, and in particular for $e=\mathbb{1}$. However, this is not true, as is shown by the example of the small one point blow up of $\mathbb{C} P^{2}$. The calculations in [17] (see also [25]) show that there is an element $Q \in \mathcal{A}_{M}$ such that $v\left(Q^{k}\right)+v\left(Q^{-k}\right) \rightarrow \infty$. (See $\$ 2$ for notation.) Further there is a constant $c$ and an element $\alpha \in \pi_{1}($ Ham) such that $\mu_{\mathbb{1}}\left(\alpha^{k}\right)=v\left(Q^{k}\right)+c k$ for all $k \in \mathbb{Z}$. But if $\mu_{\mathbb{1}}$ were a quasimorphism it would restrict to a homomorphism on the abelian subgroup $\pi_{1}(\mathrm{Ham})$ and we would have $v\left(Q^{k}\right)+v\left(Q^{-k}\right)=0$. Hence it cannot be a quasimorphism. Rather it is related to the maximum of two different quasimorphisms: since $\mathbb{1}=e_{1}+e_{2}$ where each $e_{i}$ is minimal, $\mu_{\mathbb{1}} \leq \max \left(\mu_{e_{1}}, \mu_{e_{2}}\right)$ with equality at all elements where the $\mu_{e_{i}}$ take different values; cf. equation (20) in [5].
} 
In particular, the restriction of $\mu_{e}$ to the abelian subgroup $\pi_{1}(\mathrm{Ham}) \subset \widetilde{\mathrm{Ham}}$ is a homomorphism. Therefore, $\mu_{e}$ descends to a quasimorphism on Ham exactly if it vanishes on $\pi_{1}(\mathrm{Ham})$. It has the Calabi property of [3] by construction.

There are rather few known manifolds (besides $\mathbb{C} P^{n}$ ) for which $\mathcal{A}_{M}$ contains an idempotent $e$ such that both $e \mathcal{A}_{M}$ is a field and $\mu_{e}$ descends. For these conditions work in opposite directions; we need many nontrivial Gromov-Witten invariants for $e \mathcal{A}_{M}$ to be a field, but not too many (or at least the Seidel representation of $\$ 3.1$ should be controllable) if $\mu_{e}$ is to descend. In this paper the one set of new examples with a suitable idempotent $e$ are those of Proposition 1.8 (ii). Indeed, the calculations in $[19, \S 2]$ (see also [6]) show that in this case there is an idempotent $e \in \mathcal{A}_{M}$ such that $e \mathscr{A}_{M}$ is a field, while we show here that $\mu_{e}$ descends.

However, even if $e \mathcal{A}_{M}$ is not a field, Entov-Polterovich show in $[4, \S 7]$ that $\mu_{e}$ interacts in an interesting way with the geometry of $M$. For example, it provides a lower bound for the so-called fragmentation norm ${ }^{3}\|\cdot\|_{U}$ on Ham of the form

$$
\left|\mu_{e}(\tilde{\phi} \tilde{\psi})-\mu_{e}(\tilde{\phi})-\mu_{e}(\tilde{\psi})\right| \leq K \min \left\{\|\tilde{\phi}\|_{U},\|\tilde{\psi}\|_{U}\right\}, \quad \text { for all } \tilde{\phi}, \tilde{\psi} \in \widetilde{\mathrm{Ham}},
$$

where $K$ is a constant that depends only on the open set $U$ and $U$ is assumed displaceable, i.e. there is $\psi \in \operatorname{Ham} M$ such that $U \cap \psi(U)=\emptyset$. If $e \mathcal{A}_{M}$ is a field then the quantity on LHS is bounded and $\mu_{e}$ is a quasimorphism as in (ii) above. On the other hand, if this quantity is unbounded then the fragmentation norm $\|\cdot\|_{U}$ is also unbounded on Ham. Theorems 1.1 and 1.3 allow one to transfer these results to Ham in many cases; cf. Burago-Ivanov-Polterovich [2, Example 1.24].

Remark 1.11. (i) (Properties of the spectral norm and its variants.) Consider the function $V$ on $\widetilde{\mathrm{Ham}}$ given by

$$
V(\tilde{\phi}):=c(\mathbb{1}, \tilde{\phi})+c\left(\mathbb{1}, \tilde{\phi}^{-1}\right),
$$

and the corresponding function $v$ induced on Ham:

$$
v(\phi):=\inf \{V(\tilde{\phi}) \mid \tilde{\phi} \text { lifts } \phi\} .
$$

Schwarz and Oh showed that $v$ is a conjugation invariant norm on Ham, called the spectral norm. As noticed by Entov-Polterovich [3], this norm is bounded when $M=\mathbb{C} P^{n}$ or, more generally, when $Q H_{*}(M)$ is a field with respect to suitable coefficients. ${ }^{4}$ For these hypotheses imply that $c(\mathbb{1}, \cdot)$ is an (inhomogeneous) quasimorphism on Ham, so that

$$
V(\tilde{\phi})=\left|c(\mathbb{1}, \mathrm{id})-c(\mathbb{1}, \tilde{\phi})-c\left(\mathbb{1}, \tilde{\phi}^{-1}\right)\right| \leq \text { const. }
$$

\footnotetext{
${ }^{3}$ If $U$ is an open subset of $M,\|\tilde{\phi}\|_{U}$ is defined to be the minimal number $k$ such that $\tilde{\phi}$ can be written as a product of $k$ symplectomorphisms each conjugate to an element in $\widetilde{\operatorname{Ham}}^{c}(U)$, the universal cover of the group of compactly supported Hamiltonian symplectomorphisms of $U$.

${ }^{4} \mathrm{Cf}$. Albers [1, Lemma 5.11]. In this context it matters which coefficients are used for quantum homology; compare the approaches of Ostrover [25] and Entov-Polterovich [6].
} 
Note that Ostrover's argument does not apply here since $V\left(\tilde{\psi}_{s}\right)$ remains bounded on the path $\tilde{\psi}_{s}, s \geq 0$, defined in equation (2.9).

The supremum of $v(\phi)$ for $\phi \in$ Ham is called the spectral capacity of $M$, cf. Albers [1, equation (2.47)]. Since $V(\tilde{\phi}) \geq c(\mathbb{1}, \tilde{\phi})-c(\mathrm{pt}, \tilde{\phi})>0$ for $\tilde{\phi} \neq \mathrm{id}$, this capacity can be thought of as a measure of spectral spread. When $M$ is the standard torus $T^{2 n}$ it is well known that there are (normalized) functions $H: M \rightarrow \mathbb{R}$ whose flow $\tilde{\phi}_{t}^{H}$ has the property that $V\left(\tilde{\phi}_{k}^{H}\right)=k V\left(\tilde{\phi}_{1}^{H}\right) \neq 0$; see the discussion after Question 8.7 in [4]. Since Theorem 1.3 implies that $V=v$ in this case, the spectral capacity of $T^{2 n}$ is infinite. In general the spectral capacity is poorly understood. For example, it is not known whether it is always infinite when $Q H_{*}(M)$ is very far from being a field, for example if $(M, \omega)$ is aspherical.

As we explain in the proof of Lemma $2.8, c(\mathbb{1}, \tilde{\phi}) \leq \rho^{+}\left(\tilde{\phi}^{-1}\right)$. Hence one might think of minimizing $c(\mathbb{1}, \tilde{\phi})$ and $c\left(\mathbb{1}, \tilde{\phi}^{-1}\right)$ separately over the lifts of $\phi$ as we did for the Hofer norm. However, in general this procedure gives nothing interesting since $c(\mathbb{1}, \tilde{\phi})$ certainly can be negative and may well have no lower bound over a set of lifts. Instead, one can consider the functions

$$
\bar{V}: \widetilde{\operatorname{Ham}} \rightarrow \mathbb{R}, \quad \tilde{\phi} \mapsto \bar{c}(\mathbb{1}, \tilde{\phi})+\bar{c}\left(\mathbb{1}, \tilde{\phi}^{-1}\right),
$$

and

$$
\bar{v}: \operatorname{Ham} \rightarrow \mathbb{R}, \quad \phi \mapsto \inf \{\bar{V}(\tilde{\phi}) \mid \tilde{\phi} \text { lifts } \phi\}
$$

(Thus $\bar{V}$ is a symmetrized version of the function $\mu_{\mathbb{1}}$ considered in the previous remark.) If $c(\mathbb{1}, \cdot)$ is a quasimorphism then $\bar{c}(\mathbb{1}, \cdot)$ is a homogeneous quasimorphism and hence satisfies $\bar{c}(\mathbb{1}, \tilde{\phi})=-\bar{c}\left(\mathbb{1}, \tilde{\phi}^{-1}\right)$. Therefore, in this case, $\bar{V} \equiv 0$. On the other hand, as we pointed out in Remark 1.10, $\bar{V}$ does not vanish on $\pi_{1}$ (Ham) $\subset \widetilde{\text { Ham }}$ when $M$ is a small blow up of $\mathbb{C} P^{2}$. Our remarks above imply that $T^{2 n}$ has infinite $\bar{V}$-diameter, but again very little is known about $\bar{V}$ for general $M$.

Although these variants of $v$ have some uses, they are unlikely to be (pseudo)norms, since, as we explain in Remark 2.2 (iii), they probably never have the property $m(f g) \leq m(f)+m(g)$. One might also think of replacing the class $\mathbb{1}$ by some other idempotent $e$. But it is easy to see that $v(e)>0$ for any such $e$. Hence the resulting function would not take the value 0 at the identity id $\in \widetilde{\mathrm{Ham}}$.

(ii) The proof of Corollary 1.6 compares $\rho^{+}+\rho^{-}$with the Schwarz-Oh norm $v$. Since there are $\tilde{\phi} \neq \mathbb{1}$ such that $c(\mathbb{1}, \tilde{\phi}) \leq 0$ (see equation (2.9)) this approach does not help with the other problem left open in [17], namely the question of whether $\rho^{+}$ ever vanishes on some $\phi \neq \mathbb{1}$.

Acknowledgements. Many thanks to Leonid Polterovich for very helpful comments on an earlier draft of this paper, and also to Peter Albers, Alvaro Pelayo and the referee for detailed comments that have helped improve the clarity of the exposition. 


\section{Spectral invariants}

In this section we discuss the basic properties of spectral invariants and prove Lemmas 2.7 and 2.8 concerning the Hofer diameter of Ham, as well as Proposition 1.7. We assume throughout that $M$ is closed unless explicit mention is made to the contrary.

2.1. Quantum homology. To fix notation we list some facts about the small quantum homology $Q H_{*}(M):=H_{*}(M) \otimes \Lambda$. We shall take coefficients $\Lambda:=\Lambda^{\text {univ }}\left[q, q^{-1}\right]$, where $q$ is a variable of degree 2 and $\Lambda^{\text {univ }}$ is the field ${ }^{5}$ of generalized Laurent series in $t^{-1}$ with elements

$$
\lambda=\sum_{i \geq 0} r_{i} t^{\varepsilon_{i}}, \quad r_{i}, \varepsilon_{i} \in \mathbb{R}, \varepsilon_{i}>\varepsilon_{i+1}, \varepsilon_{i} \rightarrow-\infty .
$$

Thus $\Lambda^{\text {univ }}$ has a valuation $v: \Lambda^{\text {univ }} \rightarrow[-\infty, \infty)$ given by $v(\lambda):=\max \left\{\varepsilon_{i}: r_{i} \neq 0\right\}$. Observe that $v(0)=-\infty$, and for all $\lambda, \mu \in \Lambda$

$$
v(\lambda+\mu) \leq \max (v(\lambda), v(\mu)), \quad v(\lambda \mu)=v(\lambda)+v(\mu), \quad v\left(\lambda^{-1}\right)=-v(\lambda) .
$$

This valuation extends to $Q H_{*}(M)$ in the obvious way: namely, for any $a_{i} \in H_{*}(M)$ we set

$$
v\left(\sum a_{i} \otimes q^{d_{i}} t^{\varepsilon_{i}}\right)=\max \left\{\varepsilon_{i}: a_{i} \neq 0\right\} .
$$

The quantum product $a * b$ of the elements $a, b \in H_{*}(M) \subset Q H_{*}(M)$ is defined as follows. Let $\xi_{i}, i=0, \ldots, m$, be a basis for $H_{*}(M)$ with dual basis $\left\{\xi_{i}^{* M}\right\}$. Thus

$$
\xi_{i}^{* M} \cdot M \xi_{j}=\delta_{i j} .
$$

We use this slightly awkward notation to reserve $\xi_{i}^{*}$ for later use; cf. equation (4.3). Also $\cdot M$ (which is often simplified to $\cdot$ ) denotes the intersection product

$$
H_{d}(M) \otimes H_{2 n-d}(M) \rightarrow H_{0}(M) \equiv \mathbb{R} .
$$

Further, denote by $H_{2}^{S}(M)$ the spherical homology group, i.e. the image of the Hurewicz map $\pi_{2}(M) \rightarrow H_{2}(M)$. Then

$$
a * b:=\sum_{i, \beta \in H_{2}^{S}(M)}\left\langle a, b, \xi_{i}\right\rangle_{\beta}^{M} \xi_{i}^{* M} \otimes q^{-c_{1}(\beta)} t^{-\omega(\beta)},
$$

where $\left\langle a, b, \xi_{i}\right\rangle_{\beta}^{M}$ denotes the Gromov-Witten invariant that counts curves in $M$ of class $\beta$ through the homological constraints $a, b, \xi_{i}$. Note that $\operatorname{deg}(a * b)=$

\footnotetext{
${ }^{5}$ We use the ground field $\mathbb{R}$ here since later on we use homology with $\mathbb{R}$ coefficients, but could equally well take $r_{i} \in \mathbb{C}$ as in Entov-Polterovich [5].
} 
$\operatorname{deg} a+\operatorname{deg} b-2 n$, and the identity element is $\mathbb{1}:=[M]$. The product is extended to $H_{*}(M) \otimes \Lambda$ by linearity over $\Lambda$.

Later it will be useful to consider the $\Lambda$-submodule

$$
\mathcal{Q}_{-}:=\bigoplus_{i<2 n} H_{i}(M) \otimes \Lambda \text {. }
$$

(Here as usual $2 n=\operatorname{dim} M$.) The following (easy) result was proved in [19].

Lemma 2.1. (i) If $M$ is not strongly uniruled then $Q_{-}$is an ideal in $Q H_{*}(M)$.

(ii) If $Q_{-}$is an ideal and if $u \in Q H_{2 n}(M)$ is invertible, then $u=\mathbb{1} \otimes \lambda+x$ for some nonzero $\lambda \in \Lambda$ and some $x \in Q_{-}$.

Note that if quantum multiplication is undeformed then the elements of $\mathcal{Q}_{-}$are nilpotent so that all elements $\mathbb{1} \otimes \lambda+x$ with $\lambda \neq 0$ and $x \in \mathcal{Q}_{-}$are invertible. However, if we assume only that $\mathcal{Q}_{-}$is an ideal, then an element of the form $\mathbb{1} \otimes \lambda+x$ might not be invertible. For example it could be a nontrivial idempotent. See [19] for further details.

Note. Whenever we write a unit as $\mathbb{1} \otimes \lambda+x$ we assume, unless explicit mention is made to the contrary, that $\lambda \neq 0$ and $x \in \mathcal{Q}_{-}$.

2.2. Spectral invariants and norms. One way to estimate the length of a Hamiltonian path is to use the Schwarz-Oh spectral invariants; see [27], [22] and Usher [29]. (They are also explained in [21, Chapter 12.4].) For each element $\tilde{\phi} \in \widetilde{\text { Ham }}$ and each nonzero element $a \in Q H_{*}(M)$ the number $c(a, \tilde{\phi}) \in \mathbb{R}$ has the following properties:

$$
\begin{aligned}
& -\|\tilde{\phi}\| \leq c(a, \tilde{\phi})=c\left(a, \tilde{\psi} \tilde{\phi} \tilde{\psi}^{-1}\right) \leq\|\tilde{\phi}\| \text { for } a \in H_{*}(M), \tilde{\psi} \in \widetilde{\mathrm{Ham}}, \\
& c(\lambda a, \tilde{\phi})=c(a, \tilde{\phi})+v(\lambda) \text { for all } \lambda \in \Lambda, \\
& c(a, \tilde{\phi} \circ \gamma)=c(\delta(\gamma) * a, \tilde{\phi}) \text { for all } \gamma \in \pi_{1}(\operatorname{Ham}), \\
& c(a * b, \tilde{\phi} \circ \tilde{\psi}) \leq c(a, \tilde{\phi})+c(b, \tilde{\psi}) \text { for all } a, b \in Q H_{*}(M), \tilde{\phi}, \tilde{\psi} \in \widetilde{\mathrm{Ham}} .
\end{aligned}
$$

The third property explains how these numbers depend on the path $\tilde{\phi}$. Here, the element $\delta(\gamma) \in Q H_{*}(M)$ is called the Seidel element of the loop $\gamma$ (see [28], [16]). It is an invertible element of degree $2 n=\operatorname{dim} M$ in $Q H_{*}(M)$; we give a brief definition in $\$ 3$ below. Further, (2.2) implies that $c(a, \mathrm{id})=0$ for all $a \in H_{*}(M)$, where id denotes the constant loop at the identity. Hence, for all $\gamma \in \pi_{1}$ (Ham)

$$
c(\mathbb{1}, \gamma)=c(\delta(\gamma), \text { id })=v(\&(\gamma)), \quad \bar{c}(\mathbb{1}, \gamma)=\lim _{k \rightarrow \infty} \frac{v\left(\delta\left(\gamma^{k}\right)\right)}{k} .
$$

Remark 2.2. (i) Equation (2.2) implies that the lim inf defining the asymptotic invariants $\bar{c}(a, \cdot)$ always exists. When $a^{2}=a$ standard arguments based on (2.5) show that one can replace the lim inf by an ordinary limit; cf. [3, §4.2]. 
(ii) The first two properties (2.2), (2.3) of the spectral invariants are obviously inherited by the asymptotic invariants. The third is too, because $\pi_{1}$ (Ham) lies in the center of $\widetilde{\text { Ham }}$; cf. the proof of Proposition 2.3 below. Moreover, if $\tilde{\phi}, \tilde{\psi}$ commute then the fourth property is also inherited by $\bar{c}$. (This is the basis for the discussion in [4] of partial symplectic quasi-states.)

(iii) If $e$ is an idempotent it is easy to see that $\bar{c}(e, \tilde{\phi} \tilde{\psi}) \leq c(e, \tilde{\phi})+c(e, \tilde{\psi})$ for all $\tilde{\phi}, \tilde{\psi}$. But in general the asymptotic invariants only have good algebraic properties when $e$ is an idempotent such that $e \mathcal{A}_{M}$ is a field; cf. Remark 1.10. However even in this case it is impossible to have $\bar{c}(e, \tilde{\phi} \tilde{\psi}) \leq \bar{c}(e, \tilde{\phi})+\bar{c}(e, \tilde{\psi})$ for all $\tilde{\phi}, \tilde{\psi}$. Indeed, as Polterovich points out, ${ }^{6}$ because $\bar{c}\left(e, \tilde{\phi}^{-1}\right)=-\bar{c}(e, \tilde{\phi})$ for such $e$, if this inequality did always hold one would have

$$
-\bar{c}(e, \tilde{\phi} \tilde{\psi})=\bar{c}\left(e,(\tilde{\phi} \tilde{\psi})^{-1}\right) \leq \bar{c}\left(e, \tilde{\phi}^{-1}\right)+\bar{c}\left(e, \tilde{\psi}^{-1}\right)=-\bar{c}(e, \tilde{\phi})-\bar{c}(e, \tilde{\psi}) .
$$

But then we would have equality, i.e. $\bar{c}(e, \cdot)$ would be a surjective homomorphism. It would then descend to a nontrivial homomorphism Ham $\rightarrow \mathbb{R} / \Gamma$, where $\Gamma$ is the image of the countable ${ }^{7}$ group $\pi_{1}$ (Ham). Since Ham is perfect, this is impossible. (Incidentally, this argument also shows that when $e \mathcal{A}_{M}$ is a field it is impossible that $c(e, \tilde{\phi})=\bar{c}(e, \tilde{\phi})$ for all $\tilde{\phi}$. This is obvious from the explicit calculation in formula (2.9) below. On the other hand, the current argument is structural and hence might apply in other situations as well.)

Much of following proposition is implicit in Entov-Polterovich [3].

Proposition 2.3. (i) The following conditions are equivalent:

(a) The spectral invariants $c(a, \cdot), a \in Q H_{*}(M), a \neq 0$, descend to Ham.

(b) One spectral invariant $c(a, \cdot), a \neq 0$, descends to Ham.

(c) $c(\mathbb{1}, \gamma)=v(\delta(\gamma))=0$ for all $\gamma \in \pi_{1}$ (Ham).

(d) For all $\gamma \in \pi_{1}(\mathrm{Ham}), \quad \rho(\gamma)=\mathbb{1} \otimes \lambda+x$ where $v(\lambda)=0$ and $\nu(x) \leq 0$.

(ii) The following conditions are equivalent:

(a) The asymptotic spectral invariants $\bar{c}(a, \cdot), a \in Q H_{*}(M), a \neq 0$, descend to Ham.

(b) One asymptotic spectral invariant $\bar{c}(a, \cdot), a \neq 0$, descends to Ham.

(c) For all $\gamma \in \pi_{1}$ (Ham),

$$
c(\mathbb{1}, \gamma) \geq 0, \quad \text { and } \quad \bar{c}(\mathbb{1}, \gamma)=\lim _{k \rightarrow \infty} \frac{\nu\left(8\left(\gamma^{k}\right)\right)}{k}=0 \text {. }
$$

\footnotetext{
${ }^{6}$ Private communication.
}

${ }^{7}$ A proof of this classical result is sketched in [21, Remark 9.5.6]. 
(iii) If the spectral invariants descend then so do the asymptotic invariants.

Proof. Consider (i). The equivalence of the first three conditions is immediate from (2.3) and (2.4). Part (ii) of the next lemma shows that (c) implies (d). Conversely, suppose that (d) holds, i.e. all the $\delta(\gamma)$ may be written as $\mathbb{1} \otimes \lambda+x$ where $v(\lambda)=$ $0 \geq v(x)$. Then $v(\delta(\gamma)) \leq 0$ for all $\gamma$. Moreover, if $v(\delta(\gamma))<0$ for some $\gamma$ then

$$
0=v(\mathbb{1})=v\left(\delta(\gamma) \&\left(\gamma^{-1}\right)\right) \leq v(\delta(\gamma))+v\left(\S\left(\gamma^{-1}\right)\right)<0
$$

which is a contradiction. Hence (c) holds.

Now consider (ii). If the invariants $\bar{c}$ descend then in particular we must have $\bar{c}(\mathbb{1}, \gamma)=\bar{c}(\mathbb{1}, \mathrm{id})=0$ for all $\gamma$. Further, if $c(\mathbb{1}, \gamma)=-\varepsilon<0$ then $c\left(\mathbb{1}, \gamma^{k}\right) \leq-k \varepsilon$ which implies that $\bar{c}(\mathbb{1}, \gamma)<0$. Therefore condition (c) is necessary. To prove the converse, observe that because $\gamma \in \pi_{1}$ (Ham) is in the center of $\widetilde{\mathrm{Ham}}$, the subadditivity relation (2.5) for $c$ implies that

$$
\bar{c}(a, \tilde{\phi} \circ \gamma)=\liminf \frac{1}{k}\left(c\left(a, \tilde{\phi}^{k} \gamma^{k}\right)\right) \leq \bar{c}(a, \tilde{\phi})+\bar{c}(\mathbb{1}, \gamma) .
$$

Hence if $\bar{c}(\mathbb{1}, \gamma)=0$ always,

$$
\bar{c}(a, \tilde{\phi} \circ \gamma) \leq \bar{c}(a, \tilde{\phi})=\bar{c}\left(a,(\tilde{\phi} \circ \gamma) \circ \gamma^{-1}\right) \leq \bar{c}(a, \tilde{\phi} \circ \gamma) .
$$

Thus we must always have $\bar{c}(a, \tilde{\phi} \circ \gamma)=\bar{c}(a, \tilde{\phi})$. Therefore (a) and (c) are equivalent. Since (a) implies (b) it remains to check that (b) implies (c). But the inequality

$$
\bar{c}(a, \tilde{\phi} \circ \gamma) \leq \bar{c}(a, \tilde{\phi})+\bar{c}(\mathbb{1}, \gamma)
$$

implies that $\bar{c}(\mathbb{1}, \gamma) \geq 0$, while the second part of (c) follows easily from the fact that $c\left(a, \tilde{\phi}^{k} \gamma^{k}\right)=c\left(a, \tilde{\phi}^{k}\right)+v\left(\delta\left(\gamma^{k}\right)\right)$. Thus (ii) holds. (iii) is immediate.

Lemma 2.4. (i) Every element of the form $u=\mathbb{1} \otimes \lambda+y$ with $v(\lambda) \geq v(y)$ is invertible in $Q H_{*}(M)$.

(ii) Let $u \in Q H_{*}(M)$ be an invertible element of the form $u=\mathbb{1} \otimes \mu+y$ where $\mu$ might be zero, and suppose that $v(u)=0$. Then either $v(\mu)=0=v\left(u^{-1}\right)$ or $v\left(u^{-1}\right)>0$.

Proof. First note that by the definition of the quantum product

$$
v(x * z-x \cap z) \leq v(x)+v(z)-\delta \text { for all } x, z \in Q H_{*}(M),
$$

where $\delta>0$ is the minimal energy $\omega(\beta)$ of a class $\beta \neq 0$ with nonzero GromovWitten invariant $\left\langle a_{1}, a_{2}, a_{3}\right\rangle_{\beta}$. Hence, because all elements in the undeformed ring $H_{<2 n}(M)$ are nilpotent of order $\leq n+1$,

$$
v\left(a^{n+1}\right) \leq-\delta \quad \text { for all } a \in H_{<2 n}(M) .
$$


(Here and subsequently $a^{k}$ denotes the $k$-fold quantum product $a * \cdots * a$.) Hence if $z \in Q_{-}$has $v(z) \leq 0$ then $v\left(z^{(n+1) k}\right) \leq-k \delta \rightarrow-\infty$ as $k \rightarrow \infty$. Because $v\left(z^{i}\right)$ is a nonincreasing sequence when $v(z) \leq 0$, it also diverges to $-\infty$. Thus $\sum_{i \geq 0} z^{i}$ is a well defined element of $Q H_{*}(M)$. Hence $\mathbb{1} \otimes \lambda+y$ is invertible with inverse

$$
u^{-1}:=\lambda^{-1}\left(\mathbb{1}+\lambda^{-1} y\right)^{-1}=\lambda^{-1} \sum(-1)^{i}\left(\lambda^{-1} y\right)^{i} \in Q H_{*}(M) .
$$

This proves (i).

To prove (ii) suppose that $u=\mathbb{1} \otimes \mu+y$ is a unit with $v(u)=0$. Because $\mathbb{1}$ and the components of $y$ are linearly independent, $v(u)=\max (v(\mu), v(y))$. Similarly, if we write $u^{-1}=\mathbb{1} \otimes \lambda+x$, we have $v\left(u^{-1}\right)=\max (v(\lambda), v(x))$.

If $v(\mu)=0$ then the above formula for $u^{-1}$ shows that $v\left(u^{-1}\right)=0$. So suppose that $v(\mu)<0$. Because $u u^{-1}=\mathbb{1}$, at least one of the terms $1 \otimes \lambda \mu$ and $x y$ must contain $\mathbb{1}$ with a nonzero rational coefficient. If it is the former then $v(\lambda)=-v(\mu)>0$, while if it is the latter then we must have $v(x)+v(y)>0$ so that $v(x) \geq \delta$. In either case $v\left(u^{-1}\right)>0$.

Corollary 2.5. The asymptotic spectral invariants descend to Ham if for all $\gamma$ there is $m$ such that $\delta\left(\gamma^{m}\right)=\mathbb{1} \otimes \lambda+x$ where $v(\lambda)=0$ and $x$ is nilpotent.

Proof. Suppose that $x^{N}=0$. Let $K=\max \left\{v\left(x^{k}\right) \mid 0 \leq k \leq N\right\}$. Then because 8 is a homomorphism and $v$ is subadditive

$$
v\left(8\left(\gamma^{m k}\right)\right)=v\left(\left(\delta\left(\gamma^{m}\right)\right)^{k}\right)=v\left(\lambda^{k}+k \lambda x+\ldots\right) \leq \max \{k v(\lambda), v(\lambda x), \ldots\} \leq K,
$$

for all $k \in \mathbb{Z}$ and all $\gamma$. Hence $\bar{c}\left(\mathbb{1}, \gamma^{m}\right)=0$, and hence $\bar{c}(\mathbb{1}, \gamma)=0$.

Remark 2.6. It is not hard to find conditions under which every invertible element in $Q H_{*}(M)$ has the form $\mathbb{1} \otimes \lambda+x$ where $x$ is nilpotent. For example, as we noted above, this is always true if the quantum multiplication is undeformed. For other cases see Lemma 3.2. Therefore the main difficulty in showing that the (asymptotic) spectral invariants descend lies in ensuring that the condition $v(\lambda)=0$ holds.

The numbers $c(a, \tilde{\phi})$ are defined by looking at the filtered Floer complex of the generating mean normalized Hamiltonian $H$, and turn out to be particular critical values $^{8}$ of the corresponding action functional $\mathcal{A}_{H}$; cf. Oh [23] and Usher [29]. Thus each $c(a, \tilde{\phi})$ corresponds to a particular fixed point of the endpoint $\phi_{1} \in$ Ham of $\tilde{\phi}$.

\footnotetext{
${ }^{8}$ In fact this property is essential to the existence of the spectral invariants as functions on $\widetilde{\mathrm{Ham}}$; the spectral invariants are first defined as functions on the space of Hamiltonians $H$ and one needs the spectrality property to conclude that they actually depend only on the element in $\widetilde{\text { Ham }}$ defined by the flow of $H$.
} 
It is usually very hard to calculate them. However, if $\tilde{\phi}:=\tilde{\phi}^{H}$ is generated by a $C^{2}$-small mean normalized Morse function $H: M \rightarrow \mathbb{R}$, then

$$
c\left(a, \tilde{\phi}^{H}\right)=c_{M}(a,-H), \quad a \in Q H_{*}(M),
$$

where $c_{M}(a,-H)$ are the corresponding invariants obtained from the Morse complex of $-H$. These are defined as follows. Denote by $C M_{*}(M, K)$ the usual Morse complex for the Morse function $K$. For each $\kappa \in \mathbb{R}$, it has a subcomplex $C M_{*}^{\kappa}(M, K)$ generated by the critical points $p \in \operatorname{Crit}(K)$ with critical values $K(p) \leq \kappa$. Denote by $\iota_{\kappa}$ the inclusion of the homology $H_{*}^{\kappa}$ of this subcomplex into $H_{*}^{\infty} \cong H_{*}(M)$. Then for each $a \in H_{*}(M)$

$$
c_{M}(a, K):=\inf \left\{\kappa: a \in \operatorname{Im} \iota_{\kappa}\right\} .
$$

Ostrover's construction. As pointed out by Ostrover [24], one can use the continuity properties of the $c(a, \tilde{\phi})$ to find a path $s \mapsto \tilde{\psi}_{s}$ in $\widetilde{\text { Ham }}$ whose spectral invariants tend to $\infty$ as $s \rightarrow \infty$.

Normalize $\omega$ so that $\int_{M} \omega^{n}=1$. Let $H$ be a small mean normalized Morse function, choose an open set $U$ that is displaced by $\phi_{1}^{H}$ (i.e. $\phi_{1}^{H}(U) \cap U=\emptyset$ ) and let $F: M \rightarrow \mathbb{R}$ be a function with support in $U$ and with nonzero integral $I:=\int_{M} F \omega^{n}$, so that $F-I$ is mean normalized. Denote the flow of $F$ by $f_{t}$ and consider the path $\tilde{\psi}_{s}:=\left\{f_{t s} \phi_{t}^{H}\right\}_{t \in[0,1]}$ in $\widetilde{\mathrm{Ham}}$ as $s \rightarrow \infty$. For each $s, \tilde{\psi}_{s}$ is generated by the Hamiltonian

$$
F_{s t} \# H:=s F+H \circ f_{s t} .
$$

The corresponding mean normalized Hamiltonian is

$$
K_{s}:=s F+H \circ f_{s t}-s I \text {. }
$$

By construction, $f_{s} \phi_{1}^{H}$ has the same fixed points as $\phi_{1}^{H}$, namely the critical points of $H$. Hence the continuity and spectrality properties of $c\left(a, \tilde{\psi}_{s}\right)$ imply that for each $a$ the fixed point $p_{a}$ whose critical value is $c\left(a, \tilde{\psi}_{s}\right)$ remains unchanged as $s$ increases. But the spectral value does change. In fact, if $a \in H_{*}(M)$, then when $s=0$ there is a critical point $p_{a}$ of $H$ such that $c\left(a, \tilde{\psi}_{0}\right)=c_{M}(a,-H)=-H\left(p_{a}\right)$. Hence

$$
c\left(a, \tilde{\psi}_{s}\right)=-K_{s}\left(p_{a}\right)=-H\left(p_{a}\right)+s I, \quad \text { for all } s \in \mathbb{R}, a \in H_{*}(M) .
$$

By (2.2) it follows that $\widetilde{\mathrm{Ham}}$ has infinite Hofer diameter.

The next result is well known.

Lemma 2.7. Ham has infinite diameter if either a spectral number or an asymptotic spectral number descends to Ham. 
Proof. Let $\pi: \widetilde{\mathrm{Ham}} \rightarrow$ Ham denote the projection. We will show that, for the above path $\tilde{\psi}_{s}$,

$$
\left\|\pi\left(\tilde{\psi}_{s}\right)\right\|=\left\|\psi_{s}\right\| \rightarrow \infty \quad \text { as } s \rightarrow \infty .
$$

By definition, for each $s$

$$
\left\|\psi_{s}\right\|=\inf \left\{\left\|\tilde{\psi}_{s} \circ \gamma\right\|: \gamma \in \pi_{1}(\mathrm{Ham})\right\} \geq \inf \left\{c\left(a, \tilde{\psi}_{s} \circ \gamma\right): \gamma \in \pi_{1}(\mathrm{Ham})\right\},
$$

where the inequality follows from equation (2.2). If the spectral number $c(a, \cdot)$ descends to Ham then $c\left(a, \tilde{\psi}_{s} \circ \gamma\right)=c\left(a, \tilde{\psi}_{s}\right)$ for all $\gamma$ so that the result follows from equation (2.9).

Now suppose that the asymptotic spectral number $\bar{c}(e, \cdot)$ descends to Ham for some idempotent $e$. We first claim that there are elements $\tilde{g}_{i}, i=1, \ldots, k-1$, in $\widetilde{\text { Ham }}$ that are conjugate to $\tilde{\phi}_{1}^{H}$ and such that $\tilde{\psi}_{s k} \tilde{g}_{1} \ldots \tilde{g}_{k-1}=\left(\tilde{\psi}_{s}\right)^{k}$. To see this, denote $a:=\tilde{f}_{s}, b:=\tilde{\phi}_{1}^{H}$ so that $\tilde{\psi}_{s k}=a^{k} b$ and $\left(\tilde{\psi}_{s}\right)^{k}=(a b)^{k}$. Then use the fact that

$$
\begin{aligned}
(a b)^{k} & =a^{k} b \cdot b^{-1} a^{-k} a b \ldots a b \\
& =a^{k} b \cdot b^{-1}\left(\left(a^{-k+1} b a^{k-1}\right)\left(a^{-k+2} b a^{k-2}\right) \ldots\left(a^{-1} b a\right)\right) b .
\end{aligned}
$$

Next, observe that by $(2.5)$ we have $c\left(e,(\tilde{\psi})^{k}\right) \leq k c(e, \tilde{\psi})$ for all $k>1$. Hence

$$
\bar{c}\left(e, \tilde{\psi}_{s} \circ \gamma\right)=\lim \frac{1}{k} c\left(e,\left(\tilde{\psi}_{s} \circ \gamma\right)^{k}\right) \leq c\left(e, \tilde{\psi}_{s} \circ \gamma\right) \leq\left\|\tilde{\psi}_{s} \circ \gamma\right\| .
$$

On the other hand,

$$
\begin{aligned}
\bar{c}\left(e, \tilde{\psi}_{s} \circ \gamma\right)=\bar{c}\left(e, \tilde{\psi}_{s}\right) & =\lim \frac{1}{k} c\left(e,\left(\tilde{\psi}_{s k}\right) \tilde{g}_{k-1}^{-1} \ldots \tilde{g}_{1}^{-1}\right) \\
& \geq \lim \frac{1}{k}\left(c\left(e, \tilde{\psi}_{s k}\right)-\sum_{i=1}^{k-1} c\left(e, \tilde{g}_{i}\right)\right), \\
& \geq s I-\left\|\tilde{\phi}_{1}^{H}\right\|,
\end{aligned}
$$

where the first inequality uses the identity $c(e, f g) \geq c(e, f)-c\left(e, g^{-1}\right)$ which follows from (2.5), and the second uses (2.9) and the fact that the $\tilde{g}_{i}$ are conjugate to $\tilde{\phi}_{1}^{H}$.

Lemma 2.8. The function $\rho^{+}+\rho^{-}$is unbounded on Ham whenever the asymptotic spectral numbers descend to Ham.

Proof. The proof of the inequalities in (2.2) actually shows that

$$
\int_{0}^{1} \min \left(-H_{t}\right) d t \leq c(\mathbb{1}, \tilde{\phi}) \leq \int_{0}^{1} \max \left(-H_{t}\right) d t
$$


for every mean normalized $H_{t}$ that generates $\tilde{\phi}$; see for example [21, Theorem 12.4.4]. Now suppose that $\bar{c}(\mathbb{1}, \cdot)$ descends. Equation $(2.5)$ implies that $\bar{c}\left(\mathbb{1}, \tilde{\phi}^{k}\right) \leq k c(\mathbb{1}, \tilde{\phi})$. Hence if $\tilde{\phi}$ is any lift of $\phi$,

$$
\bar{c}(\mathbb{1}, \phi)=\bar{c}(\mathbb{1}, \tilde{\phi}) \leq \int_{0}^{1} \max \left(-H_{t}\right) d t .
$$

Therefore, because $-H_{1-t}$ generates $\tilde{\phi}^{-1}$, we have $\bar{c}(\mathbb{1}, \phi) \leq \rho^{+}\left(\phi^{-1}\right)$. Applying this to the image $\psi_{s} \in$ Ham of the element $\tilde{\psi}_{s}$ of (2.9) we find that

$$
\bar{c}\left(\mathbb{1}, \psi_{s}\right)=s \leq \rho^{+}\left(\psi_{s}^{-1}\right) .
$$

Thus, because $\rho^{-} \geq 0$, the function $\rho^{+}+\rho^{-}$is not identically zero and hence is a norm. Moreover it is clearly unbounded.

Remark 2.9. As remarked in the introduction, the Hofer pseudonorm $\|\cdot\|$ is a norm on $\widehat{\mathrm{Ham}}$ if and only if it restricts to a norm on $\pi_{1}$ (Ham). The only way that I know to estimate $\|\gamma\|$ for $\gamma \in \pi_{1}(\mathrm{Ham})$ is via the spectral invariant $c(\mathbb{1}, \gamma)=\nu(\delta(\gamma))$ which is a lower bound for $\|\gamma\|$ by (2.2). If the spectral invariants descend to Ham then $c(\mathbb{1}, \gamma)=0$ for all $\gamma$ and one gets no information. For example, there are no current methods to detect the Hofer lengths of the elements of $\pi_{1}(\operatorname{Ham})$ when $(M, \omega)$ is the standard $2 n$-torus. (Of course, in this case if $n>1$ it is also unknown whether $\pi_{1}$ (Ham) itself is nonzero.) On the other hand, there are manifolds such as certain blow ups of $\mathbb{C} P^{2}$ and $S^{2}$-bundles over $S^{2}$ for which $\pi_{1}$ (Ham) is known and $v(8)$ does restrict to an injective homomorphism; see [20], [17] and the references cited therein.

We end this section by proving Proposition 1.7 about the behavior of $\rho^{+}$in the noncompact case. We need to prove:

Lemma 2.10. For each $H: M \rightarrow \mathbb{R}$ with support in $U \neq M$ there is $\delta=\delta(H, U)>$ 0 such that

$$
\rho_{U}^{+}\left(\phi_{t H}\right)=t \max H, \quad 0 \leq t \leq \delta
$$

Proof. We will prove this for open sets $U$ with smooth boundary. Since any precompact open set $U^{\prime}$ is contained in such $U$ the result for $U^{\prime}$ follows easily, since $\rho_{U^{\prime}}^{+}$is defined by taking an infimum over a smaller set than $\rho_{U}^{+}$.

Put a collar neighborhood $Y \times[-1,1] \subset M$ round the boundary of $U$ so that

$$
U \cap(Y \times[-1,1])=Y \times[-1,0) .
$$

Let $U_{\lambda}:=U \cup(Y \times[-1, \lambda])$. Choose any $\omega$-compatible almost complex structure $J_{0}$ on $M$ and choose $r>0$ so that for each $y \in Y \times\{1 / 2\}$ there is a symplectic 
embedding $f_{y}$ of the standard ball of radius $r$ into $Y \times[1 / 4,3 / 4]$ with center at $f_{y}(0)=y$. Then by monotonicity there is $\delta_{0}>0$ so that every $J_{0}$-holomorphic sphere $u: S^{2} \rightarrow U_{1}$ whose image meets both $Y \times\{1 / 4\}$ and $Y \times\{1 / 2\}$ has energy $\geq \delta_{0}$.

One condition on the constant $\delta$ is that $\delta\|H\|<\delta_{0}$, where $\|H\|=\max H-$ $\min H$. The other is that when $0<t \leq \delta$ the function $t H$ should be sufficiently small in the $C^{2}$ norm for it to be possible to embed a ball of capacity $\|t H\|$ in $R_{t H}^{-}(\varepsilon)$, the region "under the graph" of $t H$. This region is described in [17, §2.1], and the ball embedding is constructed in [15, II, Lemma 3.2].

Now let us suppose that the lemma does not hold with this choice of $\delta$. Thus if $F:=t H$ for some $t \in[0, \delta]$ we suppose that there is another Hamiltonian path $\phi_{t}^{K}$ in $\operatorname{Ham}^{c} U$ with $\phi_{1}^{K}=\phi_{1}^{F}$ that is generated by a Hamiltonian $K_{t}$ with support in $U$ and such that

$$
\int_{0}^{1} \int\left(\max _{x \in M} K_{t}\right) \omega^{n} d t<\int\left(\max _{x \in M} F\right) \omega^{n} .
$$

Under these conditions we show in $[17, \S 2]$ that there is a Hamiltonian bundle

$$
\left(U_{1}, \omega\right) \rightarrow\left(P_{1}, \Omega\right) \rightarrow S^{2}
$$

with the following properties:

(i) $P_{1}$ is trivial outside $U$. More precisely, $\left(P_{1}, \Omega\right) \rightarrow S^{2}$ contains a subbundle $(P, \Omega) \rightarrow S^{2}$ with fiber $(U, \omega)$ such that $\left(P_{1} \backslash P, \Omega\right) \rightarrow S^{2}$ may be identified with the product bundle $\left(\left(U_{1} \backslash U\right) \times S^{2}, \omega \times \alpha\right)$ where $\alpha$ is an area form on $S^{2}$.

(ii) $\int_{S^{2}} \alpha<\|F\|$.

(iii) $(P, \Omega)$ contains a symplectically embedded ball $B$ of capacity $\|F\|$.

$P_{1}$ is constructed as the union $R_{K, F}(2 \varepsilon)$ of two bundles over $D^{2}$, one a smoothing of the region in $M \times[0,1] \times \mathbb{R}$ between the graphs of $K_{t}$ and of the function $t \mapsto$ $\max K_{t}$ and with anticlockwise boundary monodromy $\left\{\phi_{t}^{K}\right\}_{t \in[0,1]}$, and the other a smoothing of the region between the graphs of $F$ and of the constant function $t \mapsto \min F$ and with clockwise boundary monodromy $\left\{\phi_{t}^{F}\right\}_{t \in[0,1]}$. The bundle is trivial outside $U$ because $F, K$ have support in $U$. Property (ii) is an immediate consequence of the construction. The region below the graph of $F$ contains the ball, which is embedded near the maximum of $F$.

Now consider the Gromov-Witten invariant that counts holomorphic spheres in $P_{1}$ in the class $\sigma=\left[p \times S^{2}\right] \in H_{2}\left(P_{1}\right)$ for $p \in U_{1} \backslash U$ and through one point $p_{0}$. If we restrict the class of allowable almost complex structures to those that are $\Omega$-tame and equal to $J_{0} \times j$ outside $P$ and choose $p_{0} \in U_{1 / 4}$, then this invariant is well defined because the energy $\Omega(\sigma)=\int_{S^{2}} \alpha$ of each curve is less than $\delta_{0}$, the amount of energy needed for a curve to enter the boundary region $P_{1} \backslash P_{1 / 2} \cong(Y \times(1 / 2,1]) \times S^{2}$. (Note that because $\left(P_{1} \backslash P, J_{0} \times j\right)$ is a product, the energy of any curve that enters 
this region is at least as much as the energy of its projection to a fiber.) We shall call this invariant $\langle\mathrm{pt}\rangle_{\sigma}^{P_{1}}$, though in principle it might depend on the product structure imposed near the boundary of $P_{1}$.

Since there is a unique $\sigma$-sphere though each point in $P_{1 / 4} \backslash P,\langle\mathrm{pt}\rangle_{\sigma}^{P_{1}}=1$. But then, by a standard argument, the nonsqueezing theorem of $[17, \S 1.4]$ holds for this fibration, i.e. every ball of radius $r$ embedded in Int $P$ has capacity $\pi r^{2} \leq \Omega(\sigma)$. But this contradicts properties (ii) and (iii) of $P_{1}$.

We conclude with a sketch proof of the nonsqueezing theorem. Let $f: B_{r} \rightarrow$ Int $P_{1}$ be a symplectic embedding of the standard $r$-ball with center $f(0)$ and image $B$. Choose an $\Omega$-tame almost complex structure $J$ on $P_{1}$ that is normalized on $P_{1} \backslash P$ and equal to $f_{*}\left(j_{n}\right)$ on $B$, where $j_{n}$ is the standard structure on $\mathbb{C}^{n}$. By hypothesis there is a $J$-holomorphic sphere $C$ in class $\sigma$ through $f(0)$, and so

$$
\Omega(\sigma)=\int_{C} \Omega>\int_{C \cap B} \Omega \geq \int_{f^{-1}(C \cap B)} \omega_{0} \geq \pi r^{2},
$$

where the last inequality holds because $f^{-1}(C \cap B)$ is a properly embedded holomorphic curve through the center of $B_{r} \subset \mathbb{C}^{n}$. For more details, see [21, Chapter 9].

Remark 2.11. Because the homology of $M$ has no fundamental class when $M$ is noncompact, it is not clear how to understand quantum homology and the Seidel representation in this case. Nevertheless, as we will see below, for the problems under consideration here we do not need to know everything about the Seidel element $\delta(\gamma)$, but just some facts about the coefficient of the fundamental class $\mathbb{1}$ in $\delta(\gamma)$. This coefficient is given by counting the number of sections of the corresponding fibration $P_{\gamma} \rightarrow S^{2}$ that go through a point, i.e. by a Gromov-Witten invariant of the form $\langle\mathrm{pt}\rangle_{\sigma}^{P_{\gamma}}$. Therefore the above argument fits naturally into the framework developed below for the closed case.

\section{The main argument}

This section explains the main ideas and proves Theorems 1.1 and 1.3 modulo some calculations of Gromov-Witten invariants that are carried out in $\$ 4$.

3.1. The Seidel representation. Our aim in this section is to prove the following result.

Proposition 3.1. (i) If $\omega$ vanishes on $\pi_{2}(M)$ then the spectral invariants descend to Ham. 
(ii) If rank $H_{2}(M)=1, n+1 \leq N<\infty$ and $(M, \omega)$ is not strongly uniruled then the spectral invariants descend. The same conclusion holds if $N \geq n-1$ and $(M, \omega)$ is negatively monotone.

(iii) If rank $H_{2}(M)=1$ and $n+1 \leq N<\infty$ then the asymptotic spectral invariants descend.

The main tool is the Seidel representation 8 . We will see that the stringent hypotheses above guarantee that the relevant moduli space of spheres has no bubbling so that it is compact. The argument goes back to Seidel ${ }^{9}$ though it was first published by Schwarz, who showed in $[27, \S 4.3]$ that the spectral invariants descend when both $\omega$ and $c_{1}$ vanish on $\pi_{2}(M)$. We generalize this result in (i) above. Part (iii) is a mild generalization of a result of Entov-Polterovich [3] who proved that the asymptotic spectral invariants descend when $M=\mathbb{C} P^{n}$.

After defining 8 , we shall calculate it under the conditions of the above proposition (see Lemma 3.2). Finally we prove the various cases of the proposition.

We shall think of the Seidel representation as a homomorphism

$$
\delta: \pi_{1}(\operatorname{Ham}(M, \omega)) \rightarrow Q H_{2 n}(M)^{\times},
$$

to the degree $2 n$ multiplicative units of the small quantum homology ring, where $2 n:=\operatorname{dim} M$. To define it, observe that each loop $\gamma=\left\{\phi_{t}\right\}$ in Ham $M$ gives rise to an $M$-bundle $P_{\gamma} \rightarrow S^{2}$ defined by the clutching function $\gamma$ :

$$
P_{\gamma}:=\left(M \times D_{+}\right) \cup\left(M \times D_{-}\right) / \sim, \quad\left(\phi_{t}(x), e^{2 \pi i t}\right)_{+} \sim\left(x, e^{2 \pi i t}\right)_{-} .
$$

(Here $D_{ \pm}$are two copies of the unit disc with union $S^{2}$.) Because the loop $\gamma$ is Hamiltonian, the fiberwise symplectic form $\omega$ extends to a closed form $\Omega$ on $P_{\gamma}$, that we can arrange to be symplectic by adding the pullback of a suitable form on the base $S^{2}$.

The bundle $P_{\gamma} \rightarrow S^{2}$ carries two canonical cohomology classes, the first Chern class $c_{1}^{\text {Vert }}$ of the vertical tangent bundle and the coupling class $u_{\gamma}$, which is the unique class that extends the fiberwise symplectic class $[\omega]$ and is such that $u_{\gamma}^{n+1}=0$. Then, with notation as in (2.1), we define

$$
\delta(\gamma):=\sum_{\sigma, i}\left\langle\xi_{i}\right\rangle_{\sigma}^{P_{\gamma}} \xi_{i}^{* M} \otimes q^{-c_{1}^{\text {Vert }}(\sigma)} t^{-u_{\gamma}(\sigma)} \in H_{*}(M) \otimes \Lambda,
$$

(cf. [21, Definition 11.4.1].) Thus $\delta(\gamma)$ is obtained by "counting" all section classes in $P_{\gamma}$ through one fiberwise constraint $\xi_{i}$. As in Seidel's original paper [28], one can also think of it as the Floer continuation map around the loop $\gamma$; cf. [21, §12.5]. We also note for later use that for all $b \in H_{*}(M)$

$$
\diamond(\gamma) * b=\sum_{\sigma, i}\left\langle b, \xi_{i}\right\rangle_{\sigma}^{P_{\gamma}} \xi_{i}^{* M} \otimes q^{-c_{1}^{\mathrm{Vert}}(\sigma)} t^{-u_{\gamma}(\sigma)},
$$

\footnotetext{
${ }^{9}$ Private communication.
} 
i.e. it is given by counting section classes with two fiberwise constraints.

We will also often use the fact that if $\sigma_{0}$ is the homology class of a section of $P_{\gamma} \rightarrow S^{2}$, then every other section in $H_{2}\left(P_{\gamma}\right)$ may be written as $\sigma_{0}+\beta$ for a unique $\beta \in H_{2}(M) \subset H_{2}\left(P_{\gamma}\right)$. Note that

$$
c_{1}^{\text {Vert }}\left(\sigma_{0}+\beta\right)=c_{1}^{\text {Vert }}\left(\sigma_{0}\right)+c_{1}(\beta)
$$

where as usual $c_{1}$ denotes $c_{1}(T M)$.

The first parts of the following lemma are due to Seidel [28].

Lemma 3.2. Let $N$ be the minimal Chern number of $M$ and $\gamma \in \pi_{1}(\operatorname{Ham} M)$.

(i) If $N \geq 2 n+1$ then $8(\gamma)=\mathbb{1} \otimes \lambda$.

(ii) If $n+1 \leq N \leq 2 n$ then $\delta\left(\gamma^{N}\right)=\mathbb{1} \otimes \lambda$. Moreover $\delta(\gamma)=\mathbb{1} \otimes \lambda$ provided that $(M, \omega)$ is not strongly uniruled.

(iii) If $N=n$ and if $(M, \omega)$ is not strongly uniruled then $\delta(\gamma)=\mathbb{1} \otimes \lambda+\mathrm{pt} \otimes q^{n} \mu$ for some $\lambda \neq 0$.

(iv) If $(M, \omega)$ is negatively monotone then $\delta(\gamma)=\mathbb{1} \otimes r_{0} t^{\varepsilon_{0}}+x$ where $x \in Q_{-}$, $v(x)<\varepsilon_{0}$.

(v) If $\omega=0$ on $\pi_{2}(M)$ then $\delta(\gamma)=(\mathbb{1}+x) \otimes r_{0} t^{\varepsilon_{0}}$ where $x \in H_{<2 n}(M)[q]$.

Proof. Suppose that $N \geq 2 n+1$ and that $\langle a, b, c\rangle_{\beta}^{M} \neq 0$, where $a, b, c \in H_{*}(M)$ have degrees $2 d_{a}, 2 d_{b}, 2 d_{c}$ respectively. Then $n+c_{1}(\beta)+d_{a}+d_{b}+d_{c}=$ $3 n$. This equation has no solution if $\left|c_{1}(\beta)\right| \geq 2 n+1$. Hence $c_{1}(\beta)=0$, and $d_{a}+d_{b}+d_{c}=2 n$. Moreover, if $a=\mathrm{pt}$ then $d_{b}=d_{c}=n$ which is impossible because $\langle\mathrm{pt}, M, M\rangle_{\beta}^{M}=0$ when $\beta \neq 0$. Hence $M$ is not strongly uniruled, and so by Lemma 2.1 all units in $Q H_{*}(M)$ have the form $\mathbb{1} \otimes \lambda+x$ where $\lambda \neq 0, x \in Q_{-}$. Therefore to prove (i) it suffices to show that $x=0$.

Next observe that the Seidel element is given by invariants of the form $\langle\xi\rangle_{\sigma}^{P_{\gamma}}$ where $\xi \in H_{*}(M)$. If $\sigma$ is a section class, then $c_{1}(T P)(\sigma)=c_{1}^{\text {Vert }}(\sigma)+2$, where the 2 is the Chern class of the tangent bundle to the section. Hence the dimension over $\mathbb{C}$ of the moduli space of parametrized $\sigma$-curves in $P_{\gamma}$ is $n+1+c_{1}^{\text {Vert }}(\sigma)+2$. Therefore the above invariant can be nonzero only if

$$
2 c_{1}^{\text {Vert }}(\sigma)+\operatorname{deg} \xi=0 .
$$

In particular, we must have $-n \leq c_{1}^{\text {Vert }}(\sigma) \leq 0$.

Now suppose that the coefficient of $\mathbb{1}$ in $\delta(\gamma)$ is nonzero. Then there is a nonzero invariant of the form $\langle\mathrm{pt}\rangle_{\sigma_{0}}^{P_{\gamma}}$, which implies that the corresponding section $\sigma_{0}$ has $c_{1}^{\text {Vert }}\left(\sigma_{0}\right)=0$. Equation (3.3) then implies that every other section $\sigma_{0}+\beta$ either has $c_{1}^{\text {Vert }}\left(\sigma_{0}+\beta\right)=0$ so that this section also contributes to the coefficient of $\mathbb{1}$, or is such 
that $c_{1}^{\text {Vert }}\left(\sigma_{0}+\beta\right)=c_{1}(\beta)$ has absolute value at least $N$. Hence if $N>n$ equation (3.4) shows that the class $\sigma_{0}+\beta$ cannot contribute to $\delta(\gamma)$. Thus $\delta(\gamma)=\mathbb{1} \otimes \lambda$. This proves (i).

In case (ii) the argument in the previous paragraph shows that all sections of $P_{\gamma}$ contributing to $\delta(\gamma)$ have the same value of $c_{1}^{\text {Vert }}$, say $-d_{\gamma}$. For short let us say that $\gamma$ is at level $d_{\gamma}$. If $d_{\gamma}>0$ then $\delta(\gamma) \in \mathcal{Q}_{-}$. Since $\delta(\gamma)$ is invertible, it follows from Lemma 2.1 that this is possible only if $(M, \omega)$ is strongly uniruled. This proves the second statement in (ii). To complete the proof of (ii) we must show that $\gamma^{N}$ always lies at level 0 even if $(M, \omega)$ is strongly uniruled.

To see this, observe that for any loops $\gamma, \gamma^{\prime}$ one can form the fibration corresponding to their product $\gamma \gamma^{\prime}$ by taking the fiber connect sum $P_{\gamma} \# P_{\gamma^{\prime}}$. Thus if $\sigma_{\gamma}$ is a section of $P_{\gamma}$ and $\sigma_{\gamma^{\prime}}$ is a section of $P_{\gamma^{\prime}}$ one can form a section of $P_{\gamma \gamma^{\prime}}$ by taking their connected sum $\sigma_{\gamma} \# \sigma_{\gamma^{\prime}}$. Under this operation the vertical Chern class adds. Thus the sections that contribute to $\delta\left(\gamma \gamma^{\prime}\right)$ either have level $d_{\gamma}+d_{\gamma^{\prime}}$ or have level $d_{\gamma}+d_{\gamma^{\prime}}-N$, depending on which of these numbers lies in the allowed range $[0, n]$. Hence if $\gamma$ lies at level $d$, for all $k \geq 1$ there is $m \geq 0$ such that $k d-m N \in[0, n]$. Since $N>n$ the only possible solution of this equation when $k=N$ is $m=d$. Therefore $\delta\left(\gamma^{N}\right)$ lies at level 0 . This proves (ii).

Almost the same argument proves (iii). Note that if there is a section at level $d>0$ then all sections that contribute to $\delta(\gamma)$ lie at this level. On the other hand if there is a section at level 0 there might also be a section at level $n$. It follows as before that $\delta(\gamma)$ must have a section at level 0 . Therefore $\delta(\gamma)=\mathbb{1} \otimes \lambda+$ pt $\otimes q^{n} \mu$. Moreover, our assumption on $Q H_{*}(M)$ implies that $\lambda \neq 0$.

To prove (iv) note that $(M, \omega)$ is not uniruled, so that by Lemma $2.18(\gamma)=$ $\mathbb{1} \otimes \lambda+x$ where $\lambda \neq 0$. Moreover, if $\sigma_{0}$ is a section class of $P$ of minimal energy with $\langle\mathrm{pt}\rangle_{\sigma} \neq 0$ then every other section class $\sigma$ with $\langle a\rangle_{\sigma} \neq 0$ for some $a \in H_{*}(M)$ has the form $\sigma=\sigma_{0}+\beta$ where $c_{1}(\beta) \leq 0$. If $c_{1}(\beta)=0$ then $\omega(\beta)=0$ and $a=$ pt. These invariants contribute to the coefficient of $t^{\varepsilon_{0}}$ in $\lambda$, where $\varepsilon_{0}=-u_{\gamma}\left(\sigma_{0}\right)$. On the other hand if $c_{1}(\beta)<0$ then $\omega(\beta)>0$ and hence these invariants contribute to terms in $x$ with valuation $<\varepsilon_{0}-\omega(\beta)$.

Finally, if $\omega$ vanishes on $\pi_{2}(M)$, then, because all Gromov-Witten invariants vanish, the quantum multiplication is undeformed. Moreover, all sections of $P_{\gamma}$ have the same energy. Hence $\delta(\gamma)=(\mathbb{1}+x) \otimes r_{0} t^{\varepsilon_{0}}$ where $0 \neq r_{0} \in \mathbb{Q}$ and $x \in \mathbb{Q}_{-}$. This proves $(\mathrm{v})$.

Definition 3.3. If $\delta(\gamma)=\mathbb{1} \otimes \lambda+x$ we define $\sigma_{0}$ to be the section class of $P_{\gamma}$ of minimal energy that contributes nontrivially to the coefficient $\lambda$. Moreover we write $\lambda=\sum_{i \geq 0} r_{i} t^{\varepsilon_{i}}$ where $r_{0} \neq 0$ and $\varepsilon_{i}>\varepsilon_{i+1}$ for all $i$.

Thus

$$
r_{0}=\langle\mathrm{pt}\rangle_{\sigma_{0}}^{P_{\gamma}}, \quad v(\lambda)=\varepsilon_{0}=-u_{\gamma}\left(\sigma_{0}\right)
$$


Note that $c_{1}^{\text {Vert }}\left(\sigma_{0}\right)=0$ by equation (3.4). The conditions in Proposition 3.1 are chosen so that the moduli space of sections in class $\sigma_{0}$ is compact. In what follows we shall often simplify notation by writing $P$ instead of $P_{\gamma}$.

Proof of Proposition 3.1. Suppose that $\left.\omega\right|_{\pi_{2}}=0$. Then $\delta(\gamma)=(\mathbb{1}+x) \otimes r_{0} t^{\varepsilon_{0}}$ by Lemma $3.2(\mathrm{v})$. We will show that $\varepsilon_{0}=0$. The claimed result then follows from Proposition 2.3 (i) (d).

Since $\left.\omega\right|_{\pi_{2}(M)}=0$ the spaces of sections that give the coefficients of $\delta(\gamma)$ are compact (when parametrized as sections) since there is no bubbling. To be more precise, note that it suffices to consider almost complex structures $J$ on $P:=P_{\gamma}$ that are compatible with the fibration $\pi: P \rightarrow S^{2}$ in the sense that $\pi$ is holomorphic and the restriction $J_{z}$ of $J$ to the fiber over $z \in S^{2}$ is $\omega$-compatible for each $z \in S^{2}$. Then all stable maps in the compactification of a space of sections consist of a section together with some bubbles in the fibers. But if $\left.\omega\right|_{\pi_{2}(M)}=0$ there are no $J_{z^{-}}$ holomorphic spheres for any $z$. Hence in this case the sections in class $\sigma_{0}$ form a compact $2 n$-dimensional manifold $\mathcal{M}:=\mathcal{M}\left(\sigma_{0}\right)$. (Here we assume that the elements of $\mathcal{M}$ are parametrized as sections.) Therefore there is a commutative diagram

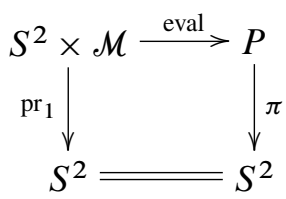

where the top arrow is the evaluation map. Moreover, $\sigma_{0}=\operatorname{eval}_{*}\left[S^{2} \times\{\mathrm{pt}\}\right]$.

Now observe that the Gromov-Witten invariant $r_{0}=\langle\mathrm{pt}\rangle_{\sigma_{0}}^{P}$ of equation (3.5) is just the degree ${ }^{10}$ of the map $\{z\} \times \mathcal{M} \rightarrow M=\pi^{-1}(z)$ that is induced by eval. Thus our hypotheses imply that eval has nonzero degree. Since the coupling class $u_{\gamma}$ satisfies $\left.u_{\gamma}\right|_{M}=[\omega]$ and $u_{\gamma}^{n+1}=0$, it easily follows that there is a class $a \in H^{2}(\mathcal{M})$ such that eval* $\left(u_{\gamma}\right)=\operatorname{pr}_{2}^{*}(a)$. Hence

$$
\varepsilon_{0}=v(\lambda)=-u_{\gamma}\left(\sigma_{0}\right)=-\int_{S^{2} \times\{\mathrm{pt}\}} \operatorname{eval}^{*} u_{\gamma}=0
$$

as required. This proves (i).

Next consider case (ii). Lemma 3.2 implies that in all cases considered here $\delta(\gamma)=\mathbb{1} \otimes \lambda+x$ where $v(x)<v(\lambda)$. Hence the spectral invariants descend provided that $v(\lambda)=0$. But for generic $\pi$-compatible $J$ on $P$ we claim that the moduli space $\mathcal{M}$ is again compact. Hence the previous argument applies to show that $v(\lambda)=0$.

To prove the claim, note that the only possible stable maps in the compactification $\overline{\mathcal{M}}$ of $\mathcal{M}$ consist of the union of a section in the class $\sigma_{0}-k \beta$ where $k>0$ together

\footnotetext{
${ }^{10}$ In fact, since $r_{0}$ must be an integer it must be \pm 1 . For its product with the corresponding integer for $\gamma^{-1}$ must be 1 .
} 
with some bubbles in classes $k_{i} \beta$. Suppose first that $c_{1}(M)=\kappa[\omega]$ where $\kappa<0$. Then because $\omega(\beta)>0, c_{1}(\beta) \leq-N$. But if $J$ is generic it induces a generic 2-parameter family $J_{z}, z \in S^{2}$, of almost complex structures on $M$. Therefore such a class $\beta$ could be represented only if $-N \geq c_{1}(\beta) \geq 2-n$, which is possible only if $N \leq n-2$. Since we assume $N \geq n-1$ there are no bubbles in this case. On the other hand if $c_{1}(M)=\kappa[\omega]$ on $\pi_{2}(M)$ where $\kappa>0$ then $c_{1}(\beta) \geq N$. Hence $c_{1}\left(\sigma_{0}-k \beta\right) \leq 2-N$. But the section is embedded and hence is regular for generic $J$. Thus for such a section to exist we must have $n+1+(2-N) \geq 3$, i.e. $n \geq N$. (This is precisely the argument in [3].) This proves (ii).

If $N \geq n+1$ but $(M, \omega)$ is strongly uniruled, then we can apply the above argument together with Corollary 2.5 to $\gamma^{N}$ to conclude that the asymptotic spectral invariants descend.

3.2. Calculating the coupling class. An essential ingredient of Schwarz's argument is that the vanishing of $\omega$ on $\pi_{2}$ implies that there is no bubbling so that the moduli space $\mathcal{M}$ is a compact manifold. One cannot replace $S^{2} \times \mathcal{M}$ here by the universal curve $\overline{\mathcal{M}}_{0,1}\left(\sigma_{0}\right)$ over some compactified virtual moduli cycle $\overline{\mathcal{M}}\left(\sigma_{0}\right)$ since the "bundle" $f: \overline{\mathcal{M}}_{0,1}\left(\sigma_{0}\right) \rightarrow \overline{\mathcal{M}}\left(\sigma_{0}\right)$ is singular over the higher strata of $\overline{\mathcal{M}}\left(\sigma_{0}\right)$. The argument we give below shows that if the relevant Gromov-Witten invariants of $M$ and $P:=P_{\gamma}$ vanish then this potential twisting does not effect eval ${ }^{*}\left(u_{\gamma}\right)$ too much, so that this class still has zero integral over the fiber of $f$. Observe that it is not enough here that the invariants of $M$ vanish; we need some control on the invariants of $P$ in either section or fiber classes.

Our reasoning is very similar to that in [16, §3]; see also [17]. There we were investigating conditions under which the ring $H^{*}(P)$ splits as a product, i.e. it is isomorphic as a ring to $H^{*}(M) \otimes H^{*}\left(S^{2}\right)$. As the next lemma makes clear, what we need here is a partial splitting of this ring.

Lemma 3.4. Suppose $\delta(\gamma)=\mathbb{1} \otimes \lambda+x$ and that there is an element $H \in H_{2 n}\left(P_{\gamma} ; \mathbb{R}\right)$ such that

(a) $H \cap[M]$ is Poincaré dual in $M$ to $[\omega]$;

(b) $H \cdot \sigma_{0}=0$;

(c) $H^{n+1}=0$.

Then $v(\lambda)=0$.

Proof. Let $u:=\operatorname{PD}_{P}(H)$, the Poincaré dual in $P:=P_{\gamma}$ to the divisor class $H$. Then (a) implies that $\left.u\right|_{M}=[\omega]$, while (c) implies that $u^{n+1}=0$. Hence $u$ is the coupling class $u_{\gamma}$. Therefore

$$
v(\lambda):=-u_{\gamma}\left(\sigma_{0}\right)=-u\left(\sigma_{0}\right)=-H \cdot \sigma_{0}=0,
$$

as required. 
It is possible that an element $H \in H_{2 n}(P)$ with the above properties exists whenever $N \geq n+1$ and $\delta(\gamma)=\mathbb{1} \otimes \lambda$. However, we can only prove this under an additional assumption ${ }^{11}$ on the structure of $Q H_{*}(M)$ that we now explain. Roughly speaking we require that the divisor classes in $H_{*}(M)$ carry all the nontrivial quantum products. To be more precise, we make the following definition.

Definition 3.5. Let $\mathcal{D}$ be the subring of $H_{\mathrm{ev}}(M)$ generated by the divisors, i.e. the elements in $H_{2 n-2}(M)$. We say that $Q H_{*}(M)$ satisfies condition (D) if $\mathscr{D}$ has an additive complement $V$ in $H_{\mathrm{ev}}(M)$ such that the following conditions hold for all $d \in \mathcal{D}, v \in V$ and $\beta \in H_{2}(M)$

$$
\text { (a) } \quad d \cdot v=0 ; \quad \text { and } \quad(b) \quad\langle d, v\rangle_{\beta}^{M}=0 \text {. }
$$

This condition is used in Lemma 4.8 in order to allow certain computations to be done recursively.

Remark 3.6. (i) This condition is trivially satisfied if either the quantum product is undeformed (since then $\langle d, v\rangle_{\beta}^{M}=0$ always) or if $\mathcal{D}=H_{\mathrm{ev}}(M)$.

(ii) When $N \geq n+1$ the only classes $\beta$ for which $\langle d, v\rangle_{\beta}^{M}$ could be nonzero have $c_{1}(\beta)=0$. (If $N=n+1$ there is one potentially nonzero invariant with two insertions and $c_{1}(\beta) \neq 0$, namely $\langle\mathrm{pt}, \mathrm{pt}\rangle_{\beta}^{M}$. But $\mathrm{pt} \in \mathscr{D}$ and so this does not affect condition (b) in Definition 3.5.)

By condition (a) in Definition 3.5 we may choose a basis $\xi_{i}, 0 \leq i \leq m$, for $H_{\text {ev }}(M)$ so that the first $m_{1}$ elements span the subring $\mathscr{D}$ while the others span $V$. Hence the first $m_{1}$ elements of the dual basis $\left\{\xi_{i}^{* M}\right\}$ will also span $\mathscr{D}$. In other words, for all $d \in \mathscr{D}$ and $\beta \in H_{2}(M)$

$$
\left\langle d, \xi_{i}\right\rangle_{\beta}^{M} \neq 0 \Longrightarrow \xi_{i}^{*}{ }^{*} \in \mathcal{D} \text { for all } i
$$

Notice also that because there is an open subset of $H_{2 n-2}(M)$ consisting of elements $D$ such that $D^{n} \neq 0$ we may assume that $\xi_{0}=\mathbb{1}$ and that for $1 \leq i \leq m_{1}$ each $\xi_{i}=D^{k}$ for some such $D$. Then each of the corresponding dual elements $\xi_{i}^{*}$ is also a sum of elements of the form $D^{k}$.

Now consider the map $s: H_{*}(M) \rightarrow H_{*+2}(P)$ defined by the identity

$$
s(a) \cdot P v:=\frac{1}{r_{0}}\langle a, v\rangle_{\sigma_{0}}^{P}, \quad v \in H_{*}(P),
$$

where $r_{0}$ is as in equation (3.5).

\footnotetext{
${ }^{11}$ This condition, though essential to the proof, is very technical and there seems no intrinsic reason why it should be necessary.
} 
Proposition 3.7. Suppose that $\delta(\gamma)=\mathbb{1} \otimes \lambda+x$, let $\sigma_{0}$ be as in Definition 3.3 and define $s$ as above. Then the element $H:=s\left(\mathrm{PD}_{M}(\omega)\right)$ satisfies the conditions of Lemma 3.4 in each of the following situations:

(i) $N \geq n+1, \delta(\gamma)=\mathbb{1} \otimes \lambda$ and $Q H_{*}(M)$ satisifies condition (D).

(ii) $N=n,(M, \omega)$ is not strongly uniruled, rank $H_{2}(M)>1$ and condition (D) holds.

(iii) $(M, \omega)$ is negatively monotone and condition (D) holds.

(iv) $Q H_{*}(M)$ is undeformed and $(M, \omega)$ is not spherically monotone with rank $H_{2}(M)=1$.

We defer the proof to $\S 4$.

Corollary 3.8. Theorems 1.1 and 1.3 hold.

Proof. Theorem 1.1 concerns the case when $Q H_{*}(M)$ is undeformed. Then $\delta(\gamma)$ always has the form $\mathbb{1} \otimes \lambda^{\prime}+x$ where $x$ is nilpotent, since these are the only invertible elements in $Q H_{*}(M)$. Moreover, if $N \geq n+1$, then $\delta(\gamma)=\mathbb{1} \otimes \lambda$ by Lemma 3.2 (i). Hence, we may suppose that the conditions of either part (i) or part (iv) of Proposition 3.7 hold. Therefore this result, together with Lemma 3.4 and Corollary 2.5, prove the theorem. Note that if $N \leq n$ we cannot conclude that the spectral invariants descend, since it is possible that $v(x)>0$ for some $\gamma$.

Similarly, part (iv) of Theorem 1.3 follows from part (iii) of Proposition 3.7. Next suppose that $N \geq n+1$ and, if $N \leq 2 n$, that $(M, \omega)$ is not strongly uniruled. Then $\delta(\gamma)=\mathbb{1} \otimes \lambda$ by Lemma 3.2. Moreover $v(\lambda)=0$ by Lemma 3.4. Hence the spectral invariants descend by part (i) of Proposition 2.3. This proves part (i) of Theorem 1.3. Parts (ii) and (iii) of this theorem follow similarly. In case (ii) we have $\delta\left(\gamma^{N}\right)=\mathbb{1} \otimes \lambda$ while in case (iii) $\delta(\gamma)=\mathbb{1} \otimes \lambda+$ pt $\otimes q^{n} \mu$. In either case $v(\lambda)=0$. Therefore the asymptotic spectral invariants descend by Corollary 2.5 .

\section{Calculations of Gromov-Witten invariants}

This section contains the proof of Proposition 3.7.

4.1. Preliminaries. We shall use the following identity of Lee-Pandharipande [12]:

$$
\operatorname{eval}_{i}^{*}(H)=\operatorname{eval}_{j}^{*}(H)+(\alpha \cdot H) \psi_{j}-\sum_{\alpha_{1}+\alpha_{2}=\alpha}\left(\alpha_{1} \cdot H\right) D_{i, \alpha_{1} \mid j, \alpha_{2}},
$$

where $\psi_{i}$ is the first Chern class of the cotangent bundle to the domain at the $i$ th marked point and $H \in H_{2 n}(P) \cong H^{2}(P)$ is any divisor. ${ }^{12}$ Lee-Pandharipande 
were working in the algebraic context and hence interpreted both sides as elements of an appropriate Picard group. Thus provided that we are working with stable maps that have at least three marked points, $D_{i, \alpha_{1} \mid j, \alpha_{2}}$ is the divisor consisting of all stable maps with two parts, one in class $\alpha_{1}$ and containing $z_{i}$ and the other in class $\alpha_{2}=\alpha-\alpha_{1}$ containing $z_{j}$.

We shall interpret (4.1) as an identity for Gromov-Witten invariants. Thus taking $i, j=1,2$ this equation states that for all classes $u, v, w \in H_{*}(P)$ and all $\alpha \in$ $\mathrm{H}_{2}(P)$,

$$
\begin{aligned}
\langle H u, v, w\rangle_{\alpha}=\langle u, H v, w\rangle_{\alpha}+(\alpha \cdot H)\langle u, \tau v, w\rangle_{\alpha} & \\
& -\sum_{j, \alpha_{1}+\alpha_{2}=\alpha}\left(\alpha_{1} \cdot H\right)\left\langle u, \eta_{j}, \ldots\right\rangle_{\alpha_{1}}\left\langle\eta_{j}^{*}, v, \ldots\right\rangle_{\alpha_{2}},
\end{aligned}
$$

where the sum is over the elements of a basis $\left\{\eta_{j}\right\}$ of $H_{*}(P)$ (with dual basis $\left\{\eta_{j}^{*}\right\}$ ) and all decompositions $\alpha_{1}+\alpha_{2}=\alpha$ of $\alpha$, and where the dots indicate that the constraint $w$ may be in either factor except if $\alpha_{2}=0$ in which case it must be in the second factor for reasons of stability. Note that $\tau$ here denotes a descendent invariant. In fact we shall only use (4.1) in cases when $H \cdot \alpha=0$ so that this term vanishes. If it does not, one should get rid of the $\tau$ insertion by using the identity $\psi_{i}=D_{i \mid j k}$, where $D_{i \mid j k}$ denotes the divisor consisting of all stable maps with two parts, one containing the point $z_{i}$ and the other containing the points $z_{j}, z_{k}$. (But often one then gets no information since the term on LHS appears in the expansion for $\tau$.) For further discussion of this point as well as a brief proof of (4.1) in the symplectic context see [19].

Notice that if we apply an identity such as (4.2) to even dimensional classes $a_{i}$ we only need to consider even dimensional $\eta_{j}, \eta_{j}^{*}$. We will make this restriction now in order to avoid irrelevant considerations of sign. Also, we will simplify the arguments that follow by choosing a basis $\left\{\eta_{j}\right\}$ for $H_{\mathrm{ev}}(P)$ of special form. We start with a basis $\xi_{i}, i \in I$, for $H_{\mathrm{ev}}(M ; \mathbb{R})$ that satisfies the condition in (3.6) and extend this to a basis for $H_{\mathrm{ev}}(P ; \mathbb{R})$ by adding elements $\xi_{j}^{*}, j \in I$, so that for all $i, j$,

$$
\xi_{i} \cdot P \xi_{j}^{*}=\delta_{i j}, \quad \xi_{i}^{*} \cdot P \xi_{j}^{*}=0 .
$$

Thus $\xi_{i}$ is a fiber class but $\xi_{j}^{*}$ is not. Note that this basis $\left\{\xi_{i}, \xi_{j}^{*}\right\}$ for $H_{\mathrm{ev}}(P)$ is self-dual. Further, $\xi_{i}^{* M}=\xi_{i}^{*} \cap M$.

With this choice of basis the sum in (4.2) breaks into two, depending on which of $\eta_{j}, \eta_{j}^{*}$ is a fiber class. To analyze the resulting product terms, we frequently use the following fact about invariants of $P$ in a fiber class $\beta$.

\footnotetext{
${ }^{12}$ Here $\alpha \cdot H$ denotes the intersection number of two homology classes $\alpha, H$ that lie in complementary degrees. If $u \in H_{i}(P)$ where $i$ is arbitrary, we shall denote by $H u$ the cap product $H \cap u \in H_{i-2}(P)$. Further, when $H=M$ is the class of the fiber, we shall write $u \cap M$ for the cap product when considered as an element in $H_{i-2}(M)$. This last distinction is not very important since $H_{*}(M)$ injects into $H_{*}(P)$ by the result of [16].
} 
Lemma 4.1. Suppose that $a, b \in H_{*}(M), v, w \in H_{*}(P)$ and $\beta \in H_{2}(M)$. Then:

(i) $\langle a, b, v\rangle_{\beta}^{P}=0$.

(ii) $\langle a, v, w\rangle_{\beta}^{P}=\langle a, v \cap M, w \cap M\rangle_{\beta}^{M}$.

Proof. This is part of [16, Proposition 1.6]. These statements hold because, as is shown in [16], one can calculate these invariants using an almost complex structure and perturbing 1-form that are compatible with the fibration $\pi: P \rightarrow S^{2}$. Hence every element in the virtual moduli cycle is represented by a curve that lies in a single fiber. (i) is then immediate, since $a, b$ can be represented in different fibers. Similarly, (ii) holds because every $\beta$-curve through $a$ must lie in the fiber containing $a$. The fact that $v \cap M, w \cap M$ do not vanish means that these cycles take care of the needed transversality normal to this fiber.

To make our argument work we also need information about certain section invariants of $P$. When $N \geq n+1$ the following lemma suffices; the proof is easy since it is based on a dimension count.

Lemma 4.2. Suppose that $N \geq n$ and that if $N=n$ then $(M, \omega)$ is not strongly uniruled. Suppose further that $\delta(\gamma)=\mathbb{1} \otimes \lambda+\mathrm{pt} \otimes q^{n} \mu$, and let $\sigma=\sigma_{0}-\beta$ where $\omega(\beta)>0$. Then for all $a \in H_{<2 n}(M)$ :

(i) For all $w \in H_{*}(P),\langle a, w, M\rangle_{\sigma}=0$ unless $c_{1}^{\text {Vert }}(\sigma)=0$ and $\operatorname{deg} w+\operatorname{deg} a=$ $2 n$.

(ii) $\langle a, b, M\rangle_{\sigma}=0$ for all $b \in H_{*}(M)$.

(iii) For all $w \in H_{*}(P),\langle a, w, M\rangle_{\sigma}$ depends only on $w \cap M$.

Proof. Statement (i) holds by a dimension count as in the proof of Lemma 3.2. (ii) holds because an invariant of this form with only two nontrivial fiber insertions is determined by $\&$; namely by (3.2) $\left\langle a, \xi_{i}\right\rangle_{\sigma}$ is the coefficient of $\xi_{i}^{* M} \otimes q^{-c_{1}^{\text {Vert }}(\sigma)} t^{-u_{\gamma}(\sigma)}$ in

$$
\S(\gamma) * a=\left(\mathbb{1} \otimes \lambda+\mathrm{pt} \otimes q^{n} \mu\right) * a=a \otimes \lambda
$$

Since $-u_{\gamma}(\sigma)>v(\lambda)$ this vanishes. Note that for this argument to apply we need that either $\mu=0$ (which happens when $N \geq n+1$ ) or pt $* a=0$, i.e. $M$ not strongly uniruled.

Statement (iii) is an immediate consequence of (ii) because any two classes $w, w^{\prime}$ with $w \cap M=w^{\prime} \cap M$ differ by $w-w^{\prime} \in H_{*}(M)$. 
4.2. Technical lemmas. This section contains two rather technical results about the vanishing of certain Gromov-Witten invariants needed for some of the cases considered by Proposition 3.7. However, they are not needed when $N \geq n+1$, and the reader might do well to read the next section first, coming back to this section later.

If $N=n$ we also need the following lemma about the fiber invariants of $P$.

Lemma 4.3. Suppose that $N=n$, that $(M, \omega)$ is not strongly uniruled, and that rank $H_{2}(M)>1$. Suppose further that $\delta(\gamma)=\mathbb{1} \otimes \lambda+$ pt $\otimes q^{n} \mu$ where $\lambda, \mu \in \Lambda^{\text {univ }}$. Then for any classes $s_{1}, s_{2} \in H_{2}(P)$ and any $\beta \in H_{2}(M),\left\langle s_{1}, s_{2}\right\rangle_{\beta}=0$.

Proof. A dimension count shows that the invariant is zero unless $c_{1}(\beta)=n$. If there are any nonzero invariants of this form, choose $\beta$ with minimal energy $\omega(\beta)$ so that $\left\langle s_{1}, s_{2}\right\rangle_{\beta} \neq 0$.

If $s_{1}, s_{2}$ are both fiber classes then the invariant vanishes by Lemma 4.1. If just one (say $s_{1}$ ) is a fiber class then the same lemma implies that the invariant equals $\left\langle s_{1}, \mathrm{pt}\right\rangle_{\beta}^{M}$ which vanishes because $M$ is not strongly uniruled. Hence $\left\langle s_{1}, s_{2}\right\rangle_{\beta}$ does not depend on the choice of section classes $s_{i}$.

We now make a specific choice of $s_{1}$. Since rank $H_{2}(M)>1$ there is $a \in H^{2}(M)$ such that $a(\beta)=0$. Let $F \in H_{2 n}(P)$ be any extension of $\operatorname{PD}_{M}(a) \in H_{2 n-2}(M)$. (Since $H_{*}(M)$ injects into $H_{*}(P)$ by [16] such a class exists.) Choose $b \in H_{2}(M)$ such that $F \cdot_{P} b=\mathrm{pt}$, and let $v \in H_{4}(P)$ be any extension of $b$. Then $v F:=$ $v \cap F \in H_{2}(P)$ is a section class of $P$. Therefore if $\sigma$ is any section class, it suffices to show that

$$
\langle v F, \sigma, H\rangle_{\beta}=0,
$$

where $H \in H_{2 n}(P)$ is chosen so that $H \cdot \beta=1$.

To prove this, apply (4.1) with $i=1, j=2$. We obtain

$$
\begin{aligned}
\langle v F, \sigma, H\rangle_{\beta}=\langle v, F \sigma, H\rangle_{\beta}+(\beta \cdot F)\langle v, \tau \sigma, H\rangle_{\beta} \\
-\sum_{i, \beta_{1}+\beta_{2}=\beta}\left(\beta_{1} \cdot F\right)\left(\left\langle v, \xi_{i}, \ldots\right\rangle_{\beta_{1}}\left\langle\xi_{i}^{*}, \sigma, \ldots\right\rangle_{\beta_{2}}\right. \\
\left.+\left\langle v, \xi_{i}^{*}, \ldots\right\rangle_{\beta_{1}}\left\langle\xi_{i}, \sigma, \ldots\right\rangle_{\beta_{2}}\right),
\end{aligned}
$$

where the dots indicate that the $H$ insertion could be in either factor. The first term in RHS vanishes because $F \sigma$ is a multiple of a point. Hence Lemma 4.1 implies that $\langle v, F \sigma, H\rangle_{\beta}=\langle v \cap M \text {, pt, } H \cap M\rangle_{\beta}^{M}$ which vanishes because $(M, \omega)$ is not uniruled. The second term on RHS vanishes since $\beta \cdot F=0$. Further in the sum neither $\beta_{1}$ nor $\beta_{2}$ is zero because of the factor $\beta_{1} \cdot F$. Since $c_{1}(\beta)=n=N$ one of the $\beta_{i}$ has $c_{1}=0$ and the other has $c_{1}=n$. (Other possibilities such as $c_{1}\left(\beta_{i}\right)=-n$ can be ruled out by dimensional considerations as in the proof of Proposition 3.1.) 
Consider the first sum. Since $\xi_{i}$ is a fiber class and $\operatorname{dim}(v \cap M)=2$ there are two possibilities; either $c_{1}\left(\beta_{1}\right)=0$ and $\operatorname{dim} \xi_{i}=2 n$ or $c_{1}\left(\beta_{1}\right)=n$ and $\operatorname{dim} \xi_{i}=0$. In the first case, $\xi_{i}^{*}$ is a section class so that $\left\langle\xi_{i}^{*}, \sigma, H\right\rangle_{\beta_{2}}=0$ by the minimality of $\omega(\beta)$. Therefore such a term does not contribute. But the second case also does not contribute because $(M, \omega)$ is not strongly uniruled. Therefore the first sum vanishes.

Now consider the second sum. Applying Lemma 4.1 again, we find that because $\xi_{i}$ is a fiber class the second factor here equals $\left\langle\xi_{i} \text {, pt, } H \cap M\right\rangle_{\beta_{2}}^{M}$. Hence it vanishes by hypothesis on $M$.

When all 3-point Gromov-Witten invariants in $M$ vanish the same argument gives the following conclusion.

Lemma 4.4. Suppose that the quantum multiplication in $M$ is undeformed and that rank $H_{2}(M)>1$. Then for all nonzero $\beta \in H_{2}(M), v \in H_{*}(P)$ and $F \in H_{2 n}(P)$ we have:

(i) $\left\langle F^{k}, v\right\rangle_{\beta}=0$.

(ii) $\langle\sigma, v\rangle_{\beta}=0$ for all section classes $\sigma$.

Proof. Consider (i). If any of $F^{k}, v$ are fiber classes then the invariant reduces to an invariant in $M$ and hence vanishes by assumption. Therefore, as above we may assume that $F \cdot \beta=0$. Now suppose that there is some nonzero invariant as in (i) and choose a minimal $k$ such that $\left\langle F^{k}, v\right\rangle_{\beta} \neq 0$. Then $k>1$ by the divisor axiom. Now choose $H$ so that $\beta \cdot H=1$ and expand $\left\langle F^{k}, v, H\right\rangle_{\beta}$ as before. The first term vanishes by the minimality of $k$ and the second since $\beta \cdot F=0$. Moreover, in the sum neither of $\beta_{1}, \beta_{2}$ vanish. Therefore, because each term in the sum has a fiber constraint in at least one of its factors the sum vanishes. This proves (i).

To prove (ii) let $F$ be any extension of the Poincaré dual to $[\omega]$. Then $F^{n} \cap M=\mathrm{pt}$. Hence $F^{n}=\sigma$ is a section class. Therefore $\langle\sigma, v\rangle_{\beta}=\left\langle F^{n}, v\right\rangle_{\beta}=0$ by (i).

4.3. Proof of Proposition 3.7. For simplicity we will now assume that $[\omega]$ is normalized so that $\int_{M} \omega^{n}=1$. Further, we set $h:=\operatorname{PD}_{M}(\omega)$ so that $h^{n}=$ pt. ${ }^{13}$ Recall that we define $s: H_{*}(M) \rightarrow H_{*+2}(P)$ by:

$$
s(a) \cdot P \quad v:=\frac{1}{r_{0}}\langle a, v, M\rangle_{\sigma_{0}}^{P}, \quad v \in H_{*}(P) .
$$

In particular, $s(M) \cdot P \mathrm{pt}=\frac{1}{r_{0}}\langle M, \mathrm{pt}, M\rangle_{\sigma_{0}}^{P}=1$ so that $s(M)=P$.

Lemma 4.5. Suppose that $\varnothing(\gamma)=\mathbb{1} \otimes \lambda+x$ where for all $b \in H_{<2 n}(M ; \mathbb{R})$ either $v(x * b)<v(\lambda)$ or $x * b=q y$ where $y \in Q H_{*}(M)$ involves only nonnegative powers of $q$. Then:

\footnotetext{
${ }^{13}$ Since $[\omega] \in H^{2}(M)$ need not be a rational class, $h \in H_{2 n-2}(M ; \mathbb{R})$. Therefore, in this section one should assume that homology groups have coefficients $\mathbb{R}$ unless otherwise indicated.
} 
(i) $s(\mathrm{pt})=\sigma_{0}$,

(ii) $s(a) \cap M=$ a for all $a \in H_{*}(M)$.

Proof. $\sigma_{0}$ was chosen so that $\langle\mathrm{pt}, M\rangle_{\sigma_{0}}^{P}=\langle\mathrm{pt}\rangle_{\sigma_{0}}^{P}=r_{0} \neq 0$. A count of dimensions shows that $\langle\mathrm{pt}, v\rangle_{\sigma_{0}}^{P} \neq 0$ only if $v$ is a divisor class. Thus for all $v \in H_{2 n}(P)$ the divisor axiom implies that

$$
s(\mathrm{pt}) \cdot v=\frac{1}{r_{0}}\langle\mathrm{pt}, v\rangle_{\sigma_{0}}^{P}=\frac{1}{r_{0}}\left(\sigma_{0} \cdot v\right)\langle\mathrm{pt}\rangle_{\sigma_{0}}^{P}=\sigma_{0} \cdot v .
$$

This proves (i).

Since we have already checked the case $a=[M]$ in (ii), it suffices to take $a \in H_{<2 n}(M)$. Observe that by equation (3.2) $\left\langle a, \xi_{i}\right\rangle_{\sigma_{0}}$ is the coefficient of $\xi_{i}^{* M} \otimes t^{\varepsilon_{0}}$ in

$$
\S(\gamma) * a=(\mathbb{1} \otimes \lambda+x) * a=a \otimes \lambda+x * a,
$$

The hypothesis on $x$ implies that $x * a$ does not contribute to this coefficient. Hence $\left\langle a, \xi_{i}\right\rangle_{\sigma_{0}}=r_{0} a \cdot M \xi_{i}$, and so

$$
r_{0}(s(a) \cap M) \cdot M \xi_{i}=r_{0} s(a) \cdot P \stackrel{\text { def }}{=}\left\langle a, \xi_{i}\right\rangle_{\sigma_{0}}=r_{0} a \cdot M \xi_{i}
$$

where the second equality holds by the definition (3.7) of $s$. Hence $s(a) \cap M=a$ as required.

There are many different cases in Proposition 3.7. In an attempt to avoid confusion we will first prove parts (i) and (ii). Thus we assume that $N \geq n$, with some further conditions when $N=n$.

Lemma 4.6. Suppose that the hypotheses of Lemma 4.2 hold, and that if $N=n$ those of Lemma 4.3 hold as well. Then $\left\langle h, \sigma_{0}, M\right\rangle_{\sigma_{0}}=0$.

Proof. Given any divisor class in $P$ extending $h$ we may add a suitable multiple of $M$ to obtain a class $K \in H_{2 n}(P)$ such that

$$
K \cap M=h, \quad K \cdot \sigma_{0}=0 .
$$

We then find by formula (4.1) that

$$
\begin{aligned}
\left\langle h, \sigma_{0}, M\right\rangle_{\sigma_{0}}=\langle & \left.K M, \sigma_{0}, M\right\rangle_{\sigma_{0}} \\
=\langle & \left\langle M, K \sigma_{0}, M\right\rangle_{\sigma_{0}}+\left(\sigma_{0} \cdot K\right)\left\langle M, \tau \sigma_{0}, M\right\rangle_{\sigma_{0}} \\
& \quad-\sum\left(\left(\sigma_{0}-\alpha\right) \cdot K\right)\left(\left\langle M, \xi_{i}, \ldots\right\rangle_{\sigma_{0}-\alpha}\left\langle\xi_{i}^{*}, \sigma_{0}, \ldots\right\rangle_{\alpha}\right. \\
& \left.+\left\langle M, \xi_{i}^{*}, \ldots\right\rangle_{\sigma_{0}-\alpha}\left\langle\xi_{i}, \sigma_{0}, \ldots\right\rangle_{\alpha}\right),
\end{aligned}
$$


where the dots indicate that the $M$ insertion could be in either factor. Note that the first two terms vanish because $K \cdot \sigma_{0}=0$. Suppose there is a nonzero contribution from the first sum. Because the first factor has two fiber constraints, Lemma 4.1 implies that $\sigma_{0}-\alpha$ must be a section class. Moreover

$$
0 \neq\left(\sigma_{0}-\alpha\right) \cdot K=-\alpha \cdot M h=\omega(\alpha) .
$$

Thus $\omega(\alpha)>0$ since there is a nonzero $\alpha$-invariant. But then

$$
\left\langle M, \xi_{i}\right\rangle_{\sigma_{0}-\alpha}=\left\langle M, \xi_{i}, M\right\rangle_{\sigma_{0}-\alpha}
$$

vanishes by Lemma 4.2 (ii) except possibly if $\operatorname{dim} \xi_{i}=2 n$. But this case can occur only if $N=n$ and $c_{1}(\alpha)=n$. But then $\xi_{i}^{*}$ is a section class, so that the second factor $\left\langle\xi_{i}^{*}, \sigma_{0}, \ldots\right\rangle_{\alpha}$ vanishes by Lemma 4.3 . Therefore in all cases this sum makes no contribution.

Now consider the second sum. Suppose first that $\beta:=\sigma_{0}-\alpha$ were a fiber class. Note that $\beta \neq 0$ because the sum is multiplied by $\beta \cdot K$. Then, because $M$ is a fiber constraint, Lemma 4.1 implies that the $\beta$ invariant is either $\left\langle M, \xi_{i}^{*} \cap M, M \cap M\right\rangle_{\beta}$, or is $\left\langle M, \xi_{i}^{*} \cap M\right\rangle_{\beta}$, which both vanish when $\beta \neq 0$ because the first marked point is not constrained. Thus the only nonzero terms have $\sigma_{0}-\alpha$ a section class, and hence have the form $\left\langle M, \xi_{i}^{*}, M\right\rangle_{\sigma_{0}-\alpha}^{P}\left\langle\xi_{i}, \mathrm{pt}\right\rangle_{\alpha}^{M}$ where $\omega(\alpha) \neq 0$ since $K \cdot \sigma_{0}=0$. Again there are two cases. If $N>n$ then Lemma 4.2 (i) implies that this can be nonzero only if $\operatorname{deg} \xi_{i}^{*}=0$. But $\xi_{i}^{*} \neq \mathrm{pt}$ since it is not a fiber class. Therefore this is impossible. On the other hand if $N=n$ then the first factor might be nonzero, but the second has to vanish since $M$ is not uniruled. Therefore in all cases the second sum vanishes as well.

Corollary 4.7. Under the conditions of the above lemma, if $H:=s(h)$, we have $H \cdot \sigma_{0}=0$.

Proof. By the definition of $s$ in (3.7), $s(h) \cdot \sigma_{0}=\left\langle h, \sigma_{0}, M\right\rangle_{\sigma_{0}}=0$.

Lemma 4.8. Suppose that $(M, \omega), \delta(\gamma)$ and $N$ satisfy the hypotheses of Lemma 4.6 and that $Q H_{*}(M)$ satisfies condition (D). Then:

(i) For all section classes $\sigma=\sigma_{0}-\beta$ where $\omega(\beta)>0$ and all $k$ we have $\left\langle F^{k}, a, M\right\rangle_{\sigma}=0$ whenever $F \in H_{2 n}(P)$ and $a \in H_{<2 n}(M)$.

(ii) $\left\langle H^{n+1-k}, h^{k}, M\right\rangle_{\sigma_{0}}=0$ for all $k$.

Proof. Consider (i). Choose $\sigma$ of minimal energy (i.e. $\omega(\beta)$ is maximal) and then the minimal $k$ so that $\left\langle F^{k}, a, M\right\rangle_{\sigma} \neq 0$ for some $a$ and $F$. Note that $k>1$ since $\langle F, a, M\rangle_{\sigma}=(F \cdot \sigma)\langle a, M\rangle_{\sigma}$ vanishes by Lemma 4.2 (ii).

Again by Lemma 4.2 (ii) we may add an arbitrary fiber class to $F^{k}$ without changing $\left\langle F^{k}, a, M\right\rangle_{\sigma}$. Hence, by replacing $F$ by $F-c M$ for suitable $c$, we may arrange 
that $F \cdot \sigma=0$. Now use (4.1) as in the previous lemma, moving $F$ from the first constraint to the second. Because $F \cdot \sigma=0$ we find as before that

$$
\begin{gathered}
\left\langle F^{k}, a, M\right\rangle_{\sigma}=\left\langle F^{k-1}, F a, M\right\rangle_{\sigma}-\sum(\alpha \cdot F)\left(\left\langle F^{k-1}, \xi_{i}, \ldots\right\rangle_{\alpha}\left\langle\xi_{i}^{*}, a, \ldots\right\rangle_{\sigma-\alpha}\right. \\
\left.+\left\langle F^{k-1}, \xi_{i}^{*}, \ldots\right\rangle_{\alpha}\left\langle\xi_{i}, a, \ldots\right\rangle_{\sigma-\alpha}\right) .
\end{gathered}
$$

The first term is zero by our choice of $k$. Consider the first sum. If $\alpha$ is a section class, then because of the factor $\alpha \cdot F$ we may assume that $\alpha \neq \sigma$. But then $\alpha$ has less energy than $\sigma$ since $\omega(\sigma-\alpha)>0$ so that the $\alpha$ invariant vanishes by the minimality of the energy of $\sigma$. So we may suppose that $\alpha$ is a fiber class, in which case the other factor is an invariant $\left\langle\xi_{i}^{*}, a, M\right\rangle_{\sigma-\alpha}$ in a section class with smaller energy than $\sigma$. We claim that condition (D) implies that the product of these factors must still vanish.

For suppose not. Then by Lemma 4.1

$$
\left\langle F^{k-1}, \xi_{i}\right\rangle_{\alpha}^{P}=\left\langle f^{k-1}, \xi_{i}\right\rangle_{\alpha}^{M} \neq 0
$$

where $f:=F \cap M$. Therefore (3.6) implies that $\xi_{i}^{{ }^{M} M}=\xi_{i}^{*} \cap M \in \mathscr{D}$. Thus $\xi_{i}^{*} \cap M$ is a linear combination of elements of the form $g^{k}, g \in H_{2 n-2}(M)$. But if we write $g=G \cap M$ for some $G \in H_{2 n}(P)$ all invariants of the form $\left\langle G^{k}, a, M\right\rangle_{\sigma-\alpha}$ vanish by the minimality condition on the energy of $\sigma$. But $\xi_{i}^{*} \cap M=G^{k} \cap M$. Hence $\left\langle\xi_{i}^{*}, a, M\right\rangle_{\sigma-\alpha}=0$ by Lemma 4.2 (iii).

Thus the first sum vanishes. Now consider the second sum. The second invariant has at least two fiber constraints. Hence $\sigma-\alpha$ must be a section class, so that the invariant vanishes by Lemma 4.2 (ii). This proves (i).

Now consider (ii). When $k=n$ the invariant is $\langle H \text {, pt, } M\rangle_{\sigma_{0}}$ which vanishes because $H \cdot \sigma_{0}=0$. Suppose that it does not vanish for all $k$ and choose the maximal $k$ for which it is nonzero. Expand $\left\langle H^{n+1-k}, h^{k}, M\right\rangle_{\sigma_{0}}$ by transferring one $H$ to the second constraint. As usual the first two terms in the expansion vanish and we obtain

$$
\begin{aligned}
\left\langle H^{n+1-k}, h^{k}, M\right\rangle_{\sigma_{0}}=- & \sum(\alpha \cdot H)\left(\left\langle H^{n-k}, \xi_{i}, \ldots\right\rangle_{\alpha}\left\langle\xi_{i}^{*}, h^{k}, \ldots\right\rangle_{\sigma_{0}-\alpha}\right. \\
& \left.+\left\langle H^{n-k}, \xi_{i}^{*}, \ldots\right\rangle_{\alpha}\left\langle\xi_{i}, h^{k}, \ldots\right\rangle_{\sigma_{0}-\alpha}\right) .
\end{aligned}
$$

Consider the first sum. If $\alpha$ is a section class then we may suppose $\alpha \neq \sigma_{0}$ because of the factor $\alpha \cdot H$. Therefore it has smaller energy than $\sigma_{0}$ so that the $\alpha$-invariant vanishes by part (i) of this lemma. On the other hand if $\alpha$ is a fiber class then as above condition (D) implies that $\xi_{i} \in \mathscr{D}$ and the second factor is a sum of terms of the form $\left\langle K^{n-k+1}, h^{k}, M\right\rangle_{\sigma}^{P}$ where $\sigma$ has less energy than $\sigma_{0}$. Therefore this factor vanishes by part (i). Therefore the first sum vanishes. But the second factor in the second sum has at least two fiber constraints. Therefore $\sigma_{0}-\alpha$ is a section class, and its energy is less than that of $\sigma_{0}$ since $\omega(\alpha) \neq 0$. Hence this factor must vanish by Lemma 4.2 (ii). Thus the RHS of the above expression vanishes. Therefore the LHS is zero also, contrary to hypothesis. The result follows. 
Corollary 4.9. If the hypotheses of Lemma 4.8 hold then $H^{n+1}=0$.

Proof. Putting $k=1$ into claim (ii) of Lemma 4.8 we find that $\left\langle h, H^{n}, M\right\rangle_{\sigma_{0}}^{P}=0$. But by equation (3.7) this is a multiple of the intersection $s(h) \cdot H^{n}$. Since $s(h)=H$, we obtain $H^{n+1}=0$.

Proof of Proposition 3.7. If $N \geq n+1$, condition (D) holds and $\delta(\gamma)=\mathbb{1} \otimes$ $\lambda$, then the hypotheses of Lemma 4.2 hold. Hence we may apply Lemmas 4.6 and 4.8. Therefore, the conditions of Lemma 3.4 hold by Corollaries 4.7 and 4.9 and Lemma 4.5. This proves (i). To prove (ii) note that the extra conditions here precisely match the conditions of Lemma 4.3 . Hence the proof goes through as before.

Now consider part (iii) of the proposition. The assumption here is that $(M, \omega)$ is negatively monotone and that condition (D) holds. We saw in Lemma 3.2 that in this case $\delta(\gamma)=\mathbb{1} \otimes r t^{\varepsilon_{0}}+x$ where $v(x)<\varepsilon_{0}$. Hence the conclusions of Lemma 4.5 hold.

Further Lemma 4.2 (ii) holds (though part (i) may not). To see this, recall that $\left\langle a, \xi_{i}^{* M}, M\right\rangle_{\sigma}$ is the coefficient of $\xi_{i} \otimes q^{-c_{1} \operatorname{Vert}(\sigma)} t^{-u_{\gamma}(\sigma)}$ in $\delta(\gamma) * a$. Because $v(x)<v(\lambda)$ in $\delta(\gamma)$, this must vanish when $-u_{\gamma}(\sigma)>-u_{\gamma}\left(\sigma_{0}\right)$. Therefore if we write $\sigma=\sigma_{0}-\beta$, the invariant vanishes when $\omega(\beta)>0$. Therefore part (iii) of this lemma also holds.

It is now easy to check that the proof of Lemma 4.6 goes through. The argument for the vanishing of the first sum needs no change (note that $c_{1}(\alpha)<0$ here so there is no exceptional case); while that for the second sum works using the fact that $(M, \omega)$ is not uniruled instead of Lemma 4.2 (i). It remains to check the proof of Lemma 4.8. But this holds as before, provided that condition (D) hold. This completes the proof of part (iii).

Finally consider part (iv). The assumption here is that $Q H_{*}(M)$ is undeformed, and if rank $H_{2}(M)=1$ that $(M, \omega)$ is not positively monotone. Since condition (D) holds when $Q H_{*}(M)$ is undeformed, the latter case follows from (i) if $c_{1}=0$ and from (iii) otherwise. Hence we may assume that rank $H_{2}(M)>1$. Therefore the conditions of Lemma 4.4 hold. Further, because $\delta(\gamma)=\mathbb{1} \otimes \lambda+x$ has degree $2 n$, all terms in $x$ have a positive coefficient of $q$. When $x * b=x \cap b$ this remains true for $x * b$. Hence the conclusions of Lemma 4.5 hold.

Next we claim that Lemmas 4.6 and 4.8 hold. To see this, we go though the proofs of these lemmas using Lemma 4.4 instead of Lemma 4.2 to show that the requisite terms vanish. Note for example that the fiber invariants in the expansion in Lemma 4.6 contain factors of the form $\left\langle\xi^{*}, \sigma\right\rangle_{\alpha}$ which vanish by Lemma 4.4 (ii). Similarly, in Lemma 4.8 we may use Lemma 4.4 (i). We do not need condition (D) because in a product such as

$$
\left\langle H^{n-k}, \xi_{i}, M\right\rangle_{\alpha}\left\langle\xi_{i}^{*}, h^{k}\right\rangle_{\sigma_{0}-\alpha}
$$


with $\alpha \in H_{2}(M)$ the first factor vanishes, and so we do not need to worry about the second factor. Hence the proof goes through as before.

\section{Examples}

We now prove Proposition 1.8. We shall calculate in the subring $Q H_{2 n}(M) \cong$ $H_{*}(M) \otimes \Lambda^{\text {univ }}$, i.e. by fixing the degree of the elements considered we can forget the coefficients $q^{i}$.

Recall the following construction from Kȩdra [11] and [18]. Let $\left(X, \omega_{X}\right)$ be a symplectic manifold. For each map $\alpha: S^{2} \rightarrow X$, let $\operatorname{gr}_{\alpha}$ be the graph $(z, \alpha(z)) \in$ $S^{2} \times X$ of $\alpha$, and let $\tau$ be an area form on $S^{2}$ of area 1. Choose a constant $\mu_{0}$ so that

$$
\Omega:=\mu_{0} \operatorname{pr}_{1}^{*}(\tau)+\operatorname{pr}_{2}^{*}(\omega)
$$

is nondegenerate on $\operatorname{gr}_{\alpha}$. Denote by $\left(\widetilde{P}, \widetilde{\Omega}_{\delta}\right)$ the $\delta$-blow up of $\left(S^{2} \times X, \Omega\right)$ along $\mathrm{gr}_{\alpha}$. (The parameter $\delta$ refers to the symplectic area of a line in the exceptional divisor.) Then the symplectic bundle $\pi: \widetilde{P} \rightarrow S^{2}$ has fiber $M:=\left(\tilde{X}, \tilde{\omega}_{\delta}\right)$ and corresponds to a Hamiltonian loop $\gamma_{\alpha} \in \pi_{1}(\operatorname{Ham}(M))$.

Lemma 5.1. Let $\left(X, \omega_{X}\right)$ be a symplectic manifold of dimension $2 n \geq 4$. Given a map $\alpha: S^{2} \rightarrow X$ define $\mu_{\alpha}:=\int_{S^{2}} \alpha^{*} \omega_{X}$ and $\ell_{\alpha}:=c_{1}^{X}(\alpha)$. Suppose that $\ell_{\alpha} \geq 0$ and that at least one of $\mu_{\alpha}, \ell_{\alpha}$ is nonzero. Then the Seidel element $8\left(\gamma_{\alpha}\right) \in Q H_{2 n}(M)$ of the loop $\gamma_{\alpha}$ defined above has the form $\mathbb{1} \otimes \lambda+x$ where $\nu(\lambda) \neq 0$ for small nonzero $\delta$.

Proof. Let $D$ be the exceptional divisor in $\widetilde{P}$. Denote the trivial section of $S^{2} \times X$ by $\sigma_{1}:=S^{2} \times\{p\}$. If $p \notin \mathrm{gr}_{\alpha}$ this lifts to a section $\tilde{\sigma}_{1}$ of $\widetilde{P} \rightarrow S^{2}$. If $\varepsilon$ denotes the class of the line in the fibers of the exceptional divisor, then every section class may be written as $\tilde{\sigma}_{1}-m \varepsilon+\beta$ where $\beta \in H_{2}^{S}(X)$. If $m=0$ then this class is pulled back from $S^{2} \times X$, and because $\widetilde{P}$ is obtained from $S^{2} \times X$ by blowing up along a (complex) curve with nonnegative Chern class, we may apply the results of $\mathrm{Hu}$ [9]. Thus for all $a \in H_{*}(X)$

$$
\langle a\rangle_{\widetilde{\sigma}_{1}+\beta}^{\widetilde{\widetilde{P}}}=\langle a\rangle_{\sigma_{1}+\beta}^{S^{2} \times X}
$$

Hence $\langle a\rangle_{\tilde{\sigma}_{1}+\beta}^{\widetilde{P}}=1$ if $\beta=0$ and vanishes otherwise.

Define $\kappa:=-u_{\gamma}\left(\tilde{\sigma}_{1}\right)$. The above argument shows that the coefficient $\lambda$ of $\mathbb{1}$ in $\delta(\gamma)$ contains the term $t^{\kappa}$. There might be other classes $\tilde{\sigma}_{1}-m \varepsilon+\beta$ that contribute to the coefficient of $\mathbb{1}$ in $\delta(\gamma)$ but these all appear with the coefficient $t^{\kappa+m \delta-\omega(\beta)}$ where $m \neq 0$. Thus $\nu(\lambda)$ either equals $\kappa$ or equals $\kappa+m \delta-\omega(\beta)$ for some $m \neq 0$ and $\beta \in H_{2}(X)$.

We now calculate $\kappa$. The coupling class $u_{\gamma}$ has the form $\widetilde{\Omega}_{\delta}+c_{\delta} \operatorname{pr}_{1}^{*} \tau$ where $c_{\delta}$ is chosen so that $\int_{\widetilde{P}} u_{\gamma}^{n+1}=0$. Further, because $\left.\widetilde{\Omega}_{\delta}\right|_{\tilde{\sigma}_{X}}=\left.\Omega\right|_{\sigma_{X}}$ by construction and 
$\int_{\sigma_{1}} \Omega=\mu_{0}$ by equation (5.1),

$$
-\kappa:=u_{\gamma}\left(\tilde{\sigma}_{1}\right)=c_{\delta}+\mu_{0} .
$$

We showed in [18] (see also [20]) that if $V:=\frac{1}{n !} \int_{X} \omega^{n}$ then

$$
\frac{1}{(n+1) !} \int_{\widetilde{P}}\left(\widetilde{\Omega}_{\delta}\right)^{n_{1}}=\mu_{0}\left(V-v_{\delta}\right)-v_{\delta}\left(\mu_{\alpha}-\frac{\ell_{\alpha}}{n+1} \delta\right),
$$

where $v_{\delta}:=\frac{\delta^{n}}{n !}$ is the volume of the ball cut out of $X$. But

$$
0=\frac{1}{(n+1) !} \int_{\widetilde{P}}\left(\widetilde{\Omega}_{\delta}+c_{\delta} \operatorname{pr}_{1}^{*} \tau\right)^{n+1}=\left(\frac{1}{(n+1) !} \int_{\widetilde{P}}\left(\widetilde{\Omega}_{\delta}\right)^{n+1}\right)+c_{\delta}\left(V-v_{\delta}\right),
$$

since $V-v_{\delta}$ is the volume of $\left(M, \tilde{\omega}_{\delta}\right)$. Therefore

$$
-\kappa:=c_{\delta}+\mu_{0}=\frac{v_{\delta}}{V-v_{\delta}}\left(\mu_{\alpha}-\frac{\ell_{\alpha}}{n+1} \delta\right) .
$$

Thus, provided that at least one of $\mu_{\alpha}, \ell_{\alpha}$ are nonzero, $\kappa$ is a rational function of $\delta$ with isolated zeros. Therefore neither $\kappa$ nor $\kappa+m \delta-\omega(\beta)$ vanish for sufficiently small $\delta \neq 0$. The result follows.

Proof of Proposition 1.8. The previous lemma proves (i), and so it remains to consider the case when $X$ has dimension 4 and $[\omega]$ and $c_{1}$ vanish on $\pi_{2}(X)$. It is shown in [18, Proposition 6.4] that under the given hypotheses on $X$ every Hamiltonian bundle $\left(M, \tilde{\omega}_{\delta}\right) \rightarrow(\widetilde{P}, \widetilde{\Omega}) \rightarrow S^{2}$ is constructed by blowing up some section $\sigma_{X}$ of some Hamiltonian bundle $(X, \omega) \rightarrow(P, \Omega) \rightarrow S^{2}$.

Let us denote by $\tilde{\gamma}_{X}$ the loop in $\pi_{1}(\operatorname{Ham} M)$ corresponding to the $M$-bundle $\widetilde{P} \rightarrow S^{2}$ and by $\gamma_{X}$ that corresponding to its blow down $P \rightarrow S^{2}$. As in the proof of Proposition 3.1, $\delta\left(\gamma_{X}\right)=r_{0} \mathbb{1}$ for all $\gamma_{X} \in \pi_{1}(\operatorname{Ham} X)$. Therefore there is at least one $^{14}$ section $\sigma_{X 0}:=\sigma_{X}+\beta$ of $P$ such that $\langle\mathrm{pt}\rangle_{\sigma_{X 0}}^{P} \neq 0$. Moreover both $c_{1}^{\text {Vert }}$ and the coupling class $u_{X}$ of $X$ vanish on the section $\sigma_{X 0}$, and hence on all other sections of $P$, in particular on $\sigma_{X}$.

The section classes in $\widetilde{P}$ have the form $\tilde{\sigma}_{X 0}-m \varepsilon+\beta$, where $\tilde{\sigma}_{X 0}$ is the lift of $\sigma_{X 0}$ and $\beta \in H_{2}^{S}(M)$. For such a class to contribute to the corresponding Seidel element $\delta(\tilde{\gamma})$ we need $-2 \leq c_{1}^{\text {Vert }}\left(\tilde{\sigma}_{X}-m \varepsilon+\beta\right)=-m \leq 0$. Moreover, since we may choose $\Omega$ so that the section $\sigma_{X}$ of $(P, \Omega)$ is symplectic, Hu's results ${ }^{15}$ imply that

$$
\langle\mathrm{pt}\rangle_{\tilde{\sigma}_{X 0}}^{\widetilde{P}}=\langle\mathrm{pt}\rangle_{\sigma_{X 0}}^{P} \neq 0 .
$$

\footnotetext{
${ }^{14}$ There may be several such since each coefficient of $\delta(\tilde{\gamma})$ is a sum of contributions from all sections with given values of $c_{1}^{\text {Vert }}$ and $\mu_{\tilde{\gamma}}$.

${ }^{15}$ We do not need to use Hu [9] here. All that matters is that the coefficient of $\mathbb{1}$ in $\delta\left(\tilde{\gamma}_{X}\right)$ is nonzero, which follows from the fact that $M$ is not uniruled.
} 
Therefore if $\kappa:=-u_{\tilde{\gamma}_{X}}\left(\tilde{\sigma}_{X 0}\right)$

$$
\delta\left(\tilde{\gamma}_{X}\right)=r_{0} \mathbb{1} \otimes t^{\kappa}+\xi \otimes t^{\kappa+\delta}+r \mathrm{pt} \otimes t^{\kappa+2 \delta}, \quad \text { where } \xi \in H_{2}(M) .
$$

We can now repeat the calculation of $\kappa$ in Lemma 5.1. All we need change is the interpretation of the constant $\mu_{0}$, which we now define to be the area $\operatorname{Vol}(P, \Omega) / \operatorname{Vol}\left(X, \omega_{X}\right)$ of the fibration $P \rightarrow S^{2}$. Hence $\kappa=0$.

Now observe that as in $[19, \S 2]$

$$
\varepsilon * \varepsilon=-\mathrm{pt}+\varepsilon \otimes t^{-\delta},
$$

and that quantum multiplication (in $M$ ) by the elements of $H_{\leq 2}(X) \subset H_{\leq 2}(M)$ is undeformed. In particular, pt $* \varepsilon=0$ so that

$$
v\left(\left(\varepsilon \otimes t^{\delta}\right)^{k}\right)=\delta, \quad k \geq 1 .
$$

Hence, if we decompose the class $\xi$ appearing in $\delta\left(\tilde{\gamma}_{X}\right)$ as $\xi=s \varepsilon+\xi^{\prime}$ for some $s \in \mathbb{Q}, \xi^{\prime} \in H_{2}(X)$, we have $\xi^{\prime} * \varepsilon=\xi^{\prime} * \mathrm{pt}=0$, and we easily find that

$$
0 \leq v\left(\delta\left(\tilde{\gamma}^{k}\right)\right) \leq 2 \delta, \quad k \geq 1
$$

Hence the asymptotic invariants descend by Proposition 2.3.

Remark 5.2. (i) It is perhaps worth pointing out that the equality in (5.2) does not always hold if you blow up along the graph of a class with $c_{1}$ negative. For example, suppose that you take $\alpha:=L$ to be the line in $X:=\mathbb{C} P^{2}$. Then it is not hard to check that $\delta\left(\gamma_{\alpha}\right)=\mathbb{1} \otimes t^{\kappa}+(L-E) \otimes t^{\kappa+\delta}$, where $E$ denotes the exceptional divisor in $M$. (The second term comes from counting sections in class $\tilde{\sigma}_{0}-E$.) Let us normalize the symplectic form on $X$ so that $\omega_{X}(L)=1$. Since $\gamma_{\alpha}^{-1}=\gamma_{-\alpha}$, we must then have

$$
\delta\left(\gamma_{-\alpha}\right)=\delta\left(\gamma_{\alpha}\right)^{-1}=\left(-E+\mathrm{pt} \otimes t^{\delta}\right) t^{1-2 \delta-\kappa} .
$$

Thus $\gamma_{\alpha}$, which is formed by blowing up along the graph of $-L$, has a Seidel element in which the coefficient of $\mathbb{1}$ vanishes. These calculations are carried out in detail in $[17, \S 5]$. Observe that $\gamma_{-\alpha}$ is three times the generator of $\pi_{1}(\operatorname{Ham} M)$ that is called $\alpha$ in [17]. Thus the element of $Q H_{*}(M)$ called $Q^{-3}$ in [17] has the form $8\left(\gamma_{\alpha}\right) t^{\kappa^{\prime}}$ for appropriate $\kappa^{\prime}$. See also [18, Remark 1.8].

(ii) One should be able to use the methods of [19] and Lai [13] to show that in the situation of Lemma 5.1 classes with $m \neq 0$ do not contribute to $8(\gamma)$. This calculation will be carried out elsewhere. 


\section{References}

[1] P. Albers, On the extrinsic topology of Lagrangian submanifolds. Internat. Math. Res. Notices 2005 (38) (2005), 2341-2371. Zbl 1126.53053 MR 2180810

[2] D. Burago, S. Ivanov and L. Polterovich, Conjugation invariant norms on groups of geometric origin. In Groups of diffeomorphisms in honor of Shigeyuki Morita on the occasion of his 60th birthday (Tokyo, 2006), ed. by R. Penner et al., Adv. Stud. in Pure Math. 52, Math. Soc. Japan, Tokyo 2008, 221-250 . Zbl 05526532

[3] M. Entov and L. Polterovich, Calabi quasimorphism and quantum homology. Internat. Math. Res. Notices 2003 (30) (2003), 1635-1676. Zbl 1047.53055 MR 1979584

[4] M. Entov and L. Polterovich, Quasi-states and symplectic intersections. Comment. Math. Helv. 81 (2006), 75-99. Zbl 1096.53052 MR 2208798

[5] M. Entov and L. Polterovich, Rigid subsets of symplectic manifolds. Compos. Math. 145 (3) (2009), 773-826. Zbl 05572299 MR 2507748

[6] M. Entov and L. Polterovich, Symplectic quasi-states and semi-simplicity of quantum homology. In Toric topology, ed. by M. Harada, Y. Karshon, M. Masuda and T. Panov, Contemp. Math. 460, Amer. Math. Soc., Providence, RI, 2008, 47-70. Zbl 1146.53066 MR 2428348

[7] M. Entov and L. Polterovich, $C^{0}$ rigidity of Poisson brackets. Internat. Math. Res. Notices 2009 (6) (2009), 1134-1158. Zbl 1163.53049 MR 2487493

[8] H. Hofer, On the topological properties of symplectic maps. Proc. Royal Soc. Edinburgh 115 (1990), 25-38. Zbl 0713.58004 MR 1059642

[9] J. Hu, Gromov-Witten invariants of blow ups along points and curves. Math. Z. 233 (2000), 709-39. Zbl 0948.53046 MR 1759269

[10] J. Hu, T.-J. Li and Yongbin Ruan, Birational cobordism invariance of uniruled symplectic manifolds. Invent. Math. 172 (2) (2008), 231-275. Zbl 1163.53055 MR 2390285

[11] J. Kędra, Evaluation fibrations and topology of symplectomorphisms. Proc. Amer. Math. Soc 133 (2005), 305-312. Zbl 1053.55011 MR 2086223

[12] Y.-P. Lee and R. Pandharipande, A reconstruction theorem in quantum cohomology and quantum $k$-theory. Amer. J. Math. 126 (2004), 1367-1379. Zbl 1080.14065 MR 2102400

[13] Hsin-Hong Lai, Gromov-Witten invariants of blow-ups along submanifolds with convex normal bundles. Geom. Topol. 13 (1) (2009), 1-48. Zbl 1159.14030 MR 2469512

[14] F. Lalonde and D. McDuff, The geometry of symplectic energy. Ann. of Math. 141 (1995), 349-371. Zbl 0829.53025 MR 1324138

[15] F. Lalonde and D. McDuff, Hofer's $L^{\infty}$ geometry: geodesics and stability, I, II. Invent. Math. 122 (1995), 1-33, 35-69. Zbl 0844.58020 Zbl 0844.58021 MR 1354953

[16] D. McDuff, Quantum homology of fibrations over $S^{2}$, Internat. J. Math. 11, (2000), 665-721. Zbl 1110.53307 MR 1780735

[17] D. McDuff, Geometric variants of the Hofer norm. J. Symplectic Geom. 1 (2002), 197-252. Zbl 1037.37033 MR 1959582

[18] D. McDuff, The symplectomorphism group of a blow up. Geom. Dedicata 132 (2008), 1-29. Zbl 1155.53055 MR 2396906 
[19] D. McDuff, Hamiltonian $S^{1}$-manifolds are uniruled. Duke Math. J. 146 (3) (2009), 449-507. MR 2484280

[20] D. McDuff, Loops in the Hamiltonian group - a survey. Preprint 2009. arXiv:math/0711.4086

[21] D. McDuff and D. Salamon, J-holomorphic curves and symplectic topology. Colloquium Publications 52, Amer. Math. Soc., Providence, RI, 2004. Zbl 1064.53051 MR 2045629

[22] Yong-Geun Oh, Spectral invariants, analysis of the Floer moduli spaces and geometry of the Hamiltonian diffeomorphism group. Duke Math J. 130 (2005), 199-295. Zbl 1113.53054 MR 2181090

[23] Yong-Geun Oh, Floer mini-max theory, the Cerf diagram and the spectral invariants,. J. Korean Math. Soc. 46 (2) (2009), 363-447. Zbl 05567738 MR 2494501

[24] Y. Ostrover, A comparison of Hofer's metrics on Hamiltonian diffeomorphisms and Lagrangian submanifolds. Commun. Contemp. Math 5 (2003) 803-811. Zbl 1053.53060 MR 2017719

[25] Y. Ostrover, Calabi quasi-morphisms for some non-monotone symplectic manifolds. Algebr. Geom. Topol. 6 (2006), 405-434. Zbl 1114.53070 MR 2220683

[26] L. Polterovich, Hofer's diameter and Lagrangian intersections. Internat. Math. Res. Notices 1998 (4) (1998), 217-223. Zbl 0939.37030 MR 1609620

[27] M. Schwarz, On the action spectrum for closed symplectially aspherical manifolds. Pacific J. Math 193 (2000), 419-461. Zbl 1023.57020 MR 1755825

[28] P. Seidel, $\pi_{1}$ of symplectic automorphism groups and invertibles in quantum cohomology rings. Geom. Funct. Anal. 7 (1997), 1046 -1095. Zbl 0928.53042 MR 1487754

[29] M. Usher, Spectral numbers in Floer theories. Compos. Math. 144 (6) (2008), 1581-1592. Zbl 2474322 MR 1151.53074

Received January 28, 2008

Dusa McDuff, Department of Mathematics, Barnard College, Columbia University, New York, NY 10027-6598, U.S.A.

E-mail: dmcduff@barnard.edu 